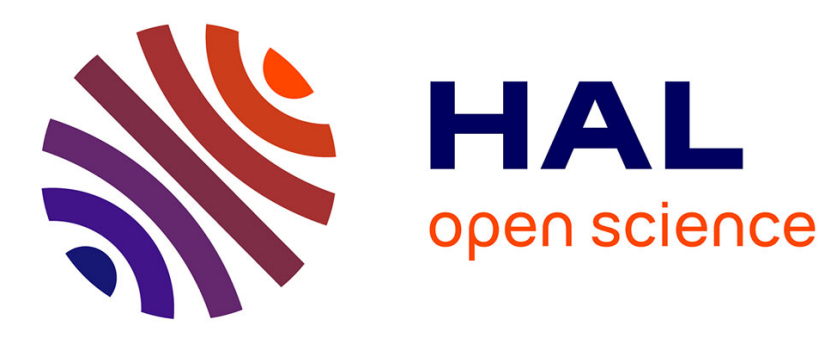

\title{
High-order IMEX-spectral schemes for computing the dynamics of systems of nonlinear Schrödinger /Gross-Pitaevskii equations
}

\author{
Xavier Antoine, Christophe Besse, Vittorio Rispoli
}

\section{To cite this version:}

Xavier Antoine, Christophe Besse, Vittorio Rispoli. High-order IMEX-spectral schemes for computing the dynamics of systems of nonlinear Schrödinger /Gross-Pitaevskii equations. Journal of Computational Physics, 2016, 327, pp.252-269. 10.1016/j.jcp.2016.09.020 . hal-01271463

\section{HAL Id: hal-01271463 \\ https://hal.science/hal-01271463}

Submitted on 9 Feb 2016

HAL is a multi-disciplinary open access archive for the deposit and dissemination of scientific research documents, whether they are published or not. The documents may come from teaching and research institutions in France or abroad, or from public or private research centers.
L'archive ouverte pluridisciplinaire HAL, est destinée au dépôt et à la diffusion de documents scientifiques de niveau recherche, publiés ou non, émanant des établissements d'enseignement et de recherche français ou étrangers, des laboratoires publics ou privés. 


\title{
High-order IMEX-spectral schemes for computing the dynamics of systems of nonlinear Schrödinger/Gross-Pitaevskii equations
}

\author{
Xavier ANTOINE*, Christophe BESSE $\dagger^{\prime}$ Vittorio RISPOLI ${ }^{\dagger}$
}

\begin{abstract}
The aim of this paper is to build and validate some explicit high-order schemes, both in space and time, for simulating the dynamics of systems of nonlinear Schrödinger/Gross-Pitaevskii equations. The method is based on the combination of high-order IMplicit-EXplicit (IMEX) schemes in time and Fourier pseudo-spectral approximations in space. The resulting IMEXSP schemes are highly accurate, efficient and easy to implement. They are also robust when used in conjunction with an adaptive time stepping strategy and appear as an interesting alternative to time-splitting pseudo-spectral (TSSP) schemes. Finally, a complete numerical study is developed to investigate the properties of the IMEXSP schemes, in comparison with TSSP schemes, for one- and two-components systems of Gross-Pitaevskii equations.
\end{abstract}

Keywords: Nonlinear Schrödinger equation; Gross-Pitaevskii equation; Bose-Einstein Condensates; spin-orbit; dynamics; high-order discretization; IMplicit-EXplicit schemes; pseudo-spectral schemes; time-splitting; adaptive time stepping

\section{Contents}

\section{Introduction}

2 High-order IMEX pseudo-spectral schemes for multi-components GPE

2.1 High-order time discretization based on IMEX-RK schemes . . . . . . . . . . . . . . 3

2.2 Adaptive time step strategy for the IMEX schemes . . . . . . . . . . . . . . . 5

2.3 Fourier pseudo-spectral discretization in space . . . . . . . . . . . . . . . . 6

2.4 Time-splitting discretizations . . . . . . . . . . . . . . . . . . 8

3 Numerical results 9

3.1 Example 1: the one-dimensional cubic NLSE . . . . . . . . . . . . . . . . . 11

3.2 Example 2: a NLSE with time-dependent potential and cubic-plus-quintic nonlinearity . . . . . . 11

3.3 Example 3: the two-components Rashba spin-orbit coupled system (1) . . . . . . . . . . . . 14

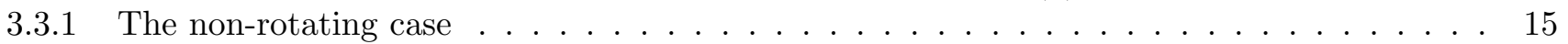

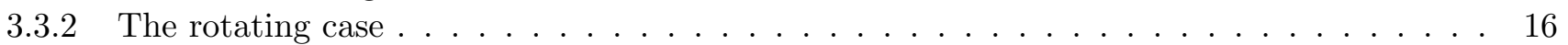

${ }^{*}$ Institut Elie Cartan de Lorraine, Université de Lorraine, UMR 7502, Inria Nancy-Grand Est (SPHINX Team), F-54506 Vandoeuvre-lès-Nancy Cedex, France.

Email: xavier.antoine@univ-lorraine.fr

${ }^{\dagger}$ Institut de Mathématiques de Toulouse, UMR CNRS 5219, Université Paul Sabatier Toulouse 3, 118 Route de Narbonne, 31062 Toulouse Cedex 9, France.

Email: christophe.besse@math.univ-toulouse.fr; vittorio.rispoli@math.univ-toulouse.fr 


\section{Introduction}

The goal of this paper is to derive high-order IMplicit-EXplicit (IMEX) pseudo-spectral schemes for general systems of NonLinear Schrödinger/Gross-Pitaevskii Equations (NLSE/GPE). In the presentation, we detail the specific case of a system of GPE that models a two-components non-rotating/rotating spin-orbit coupled Bose-Einstein Condensate (BEC) [1, 2, 10, 24, 26, 30, 32, 35, 37. However, all the developments can be directly extended to any one- or multi-components systems of GPE. More precisely, we consider the following (dimensionless) system, for $j=1,2$,

$$
i \partial_{t} \psi_{j}=\left(-\frac{1}{2} \Delta+V_{j}(\mathbf{x})+f_{j}\left(\psi_{1}, \psi_{2}\right)-\Omega L_{z}\right) \psi_{j}-\kappa R_{j} \psi_{3-j}-\lambda \psi_{3-j},
$$

where $\mathbf{x} \in \mathbb{R}^{d}$ (with $d=1,2$ or 3 and $\mathbf{x}=(x, y, z)$, correspondingly) is the space variable and $t \geq 0$ is the time variable. The wave functions for the two species are $\psi_{1,2}=\psi_{1,2}(t, \mathbf{x}) \in \mathbb{C}$. The operator $\Delta$ is the Laplace operator. The external potentials are $V_{1,2}$ and the terms $f_{1,2}\left(\psi_{1}, \psi_{2}\right)$ describe the nonlinear interactions. For example, a standard case is to consider harmonic potentials

$$
V_{j}(x, y)=\frac{1}{2}\left(\gamma_{j, x}^{2} x^{2}+\gamma_{j, y}^{2} y^{2}\right),
$$

with $\gamma_{j, x y} \in \mathbb{R}$, and cubic nonlinearities

$$
f_{j}\left(\psi_{1}, \psi_{2}\right)=\beta_{j 1}\left|\psi_{1}\right|^{2}+\beta_{j 2}\left|\psi_{2}\right|^{2},
$$

for $j=1,2$, with real-valued parameters $\beta_{11}=\beta_{22}, \beta_{12}=\beta_{21}$. The angular momentum is $L_{z}=-i\left(x \partial_{y}-y \partial_{x}\right)$, with $\Omega$ the rotational velocity. The operators $R_{j}=i \partial_{x}+(-1)^{3-j} \partial_{y}, j=1,2$, model the Rashba spin-orbit coupling effect, $\kappa$ being the Rashba spin-orbit interaction strength. This parameter is the same in both the $x$ and $y$-directions. The contribution related to $\lambda \in \mathbb{R}$ concerns a simple local coupling effect. The initial states $\psi_{j}(0, \mathbf{x})=\psi_{0 j}(\mathbf{x}), j=1,2$, are finally given. For a description of the physical model for $\lambda=0$ and a cubic nonlinearity, also better known as Rashba spin-orbit coupling model with rotation, we refer e.g. to [1]. The case without Rashba coupling is studied e.g. in [31. We assume, even if not necessary, that the rotation speed $\Omega$ and the spin-orbit interaction strength $\kappa$ are the same for the two species and that $\beta_{11}=\beta_{22}$, this last condition being required by the spin-orbit model [1].

Some macroscopic quantities are of first importance for system $(1)$ : the $L^{2}\left(\mathbb{R}^{d}\right)$-norm of the total distribution

$$
N_{\psi_{1}, \psi_{2}}(t)=\sum_{j=1}^{2} N_{\psi_{j}}(t)=\sum_{j=1}^{2}\left\|\psi_{j}\right\|_{2}^{2}=\sum_{j=1}^{2} \int_{\mathbb{R}^{d}}\left|\psi_{j}\right|^{2} d \mathbf{x}
$$

also called mass, and the energy

$$
E_{\psi_{1}, \psi_{2}}(t)=\int_{\mathbb{R}^{d}} \sum_{j=1}^{2}\left(\frac{1}{2}\left\|\nabla \psi_{j}\right\|^{2}+V_{j}\left|\psi_{j}\right|^{2}+F_{j}\left(\psi_{1}, \psi_{2}\right)-\Omega \psi_{j}^{*} L_{z} \psi_{j}-\kappa \psi_{j}^{*} R_{j} \psi_{3-j}\right)-2 \lambda \Re\left(\psi_{1} \psi_{2}^{*}\right) d \mathbf{x},
$$

where $F_{j}$ is defined by $\partial_{\psi_{j}^{*}} F_{j}=f_{j}\left(\psi_{1}, \psi_{2}\right) \psi_{j}, j=1,2$. For the cubic nonlinearity, for instance, we obtain

$$
F_{1}\left(\psi_{1}, \psi_{2}\right)=\frac{1}{2} \beta_{11}\left|\psi_{1}\right|^{4}+\frac{1}{2} \beta_{12}\left|\psi_{1}\right|^{2}\left|\psi_{2}\right|^{2}, \quad F_{2}\left(\psi_{1}, \psi_{2}\right)=\frac{1}{2} \beta_{12}\left|\psi_{1}\right|^{2}\left|\psi_{2}\right|^{2}+\frac{1}{2} \beta_{22}\left|\psi_{2}\right|^{4} .
$$

For an integer $m \geq 1$ and for $f(\psi)=|\psi|^{2 m} \psi$, we have: $F(\psi)=|\psi|^{2(m+1)} /(m+1)$. The ability of a numerical scheme to reproduce these quantities is extremely important, most particularly when they are conserved.

Deriving accurate and efficient numerical schemes which are well-adapted to describe the physics of BEC through GPE is a challenging computational problem. For the dynamics, some second-order discrete schemes in time have been developed for systems of NLSE/GPE [3, 6, 7, 10]. Among the most important methods, let us mention the (implicit) Crank-Nicolson, the (implicit) relaxation and the (explicit) time-splitting schemes which present some of the following very attractive features: unconditional stability, time transverse invariance, 
time reversibility, mass/energy conservation, dispersion relations. In addition, these schemes can be coupled to high-order and efficient spatial discretizations like pseudo-spectral FFT-based approximation schemes [3, 6. 17, 10, 11, 31, resulting in powerful numerical methods. Nevertheless, some limitations may arise when a high-order of accuracy is required in time while preserving a high precision in space. Indeed, in some important situations, deriving high-order extensions of the standard schemes, and most particularly the explicit timesplitting methods, is nontrivial when for instance the system of GPE is non-autonomous or when it cannot be reduced to a simplified situation where usual methods apply. For this last point, we refer e.g. to the recent and very interesting technique developed in [11] which makes use of rotating Lagrangian coordinate to avoid the evaluation of the angular momentum term. As a consequence, second-order time-splitting spectral schemes can be adapted [11] but going to higher-order schemes is non trivial since the system is non-autonomous. However, such a method cannot always be applied and then the full system of GPE must be discretized as for instance for system (1).

The aim of this paper is to present an alternative approach to high-order (both in space and time) timesplitting schemes which can be simply and efficiently applied to very general systems of GPE while remaining explicit. To this end, we consider high-order IMplicit-EXplicit (IMEX) time integrators based on Runge-Kutta (RK)-type discretizations schemes with adaptive time stepping techniques combined with FFT-based pseudospectral approximation methods. The resulting schemes, called IMEXSP, can easily be applied to any system of GPE. They are highly accurate, robust and despite their implicit nature they can practically be explicitly implemented and are thus also efficient. These schemes do not exactly preserve the mass nor the energy of the system but numerically fulfill these properties with a very high precision. Consequently, IMEXSP discretization schemes can be considered as very interesting alternatives to time-splitting pseudo-spectral schemes.

The paper is organized as follows. Section 2 presents the derivation of the high-order IMEXSP schemes for the multi-components GPE with adaptive time stepping technique. For completeness and comparison, subsection 2.4 discusses the time-splitting pseudo-spectral schemes that will be used in the sequel of the paper. Section 3 presents a thorough numerical study of the IMEXSP schemes for three situations: the one-dimensional cubic NLSE (subsection 3.1), the NLSE with cubic-plus-quintic nonlinearity and time-dependent potential (subsection 3.2) and finally the full two-components Rashba spin-orbit coupled system (1). Finally, we end the paper by a conclusion in section 4.

\section{High-order IMEX pseudo-spectral schemes for multi-components GPE}

\subsection{High-order time discretization based on IMEX-RK schemes}

Let us consider a non-autonomous scalar ODE

$$
\partial_{t} \psi=f(t, \psi),
$$

and a uniform time discretization $t^{0}, t^{1}, \ldots$, with constant time step $\Delta t>0$. For $m=1, \ldots, \nu$, a $\nu$-stages RK scheme and the associated numerical solution $\psi^{n+1}$ from $\psi^{n}$ are given by

$$
\begin{aligned}
& K^{m}=\psi^{n}+\Delta t \sum_{\ell=1}^{\nu} a_{m \ell} f\left(t^{n \ell}, K^{\ell}\right), \\
& \psi^{n+1}=\psi^{n}+\Delta t \sum_{\ell=1}^{\nu} w_{\ell} f\left(t^{n \ell}, K^{\ell}\right),
\end{aligned}
$$

with the weighted times $t^{n \ell}:=t^{n}+c_{\ell} \Delta t$. The real-valued parameters $a_{m \ell}, c_{\ell}$ and $w_{\ell}($ for $m, \ell=1, \ldots, \nu)$ are suitably chosen to guarantee some interesting properties (i.e. consistency, stability, high-order of accuracy, conservation, time reversibility, etc.) of the scheme [17, 18]. A RK scheme is explicit if and only if $a_{m \ell}=0$, for $m \leq \ell$, (i.e. the associated matrix $A:=\left(a_{m \ell}\right)_{1 \leq m, \ell \leq \nu}$ is strictly lower triangular), otherwise it is implicit. A standard way to represent a ( $\nu$-stages) RK scheme is through a Butcher tableau. If we introduce the two vectors $\mathbf{c}:=\left(c_{\ell}\right)_{1 \leq \ell \leq \nu}$ and $\mathbf{w}:=\left(w_{\ell}\right)_{1 \leq \ell \leq \nu}$ in $\mathbb{R}^{\nu}$ and the matrix $A \in \mathbb{R}^{\nu \times \nu}$, then (all the coefficients of) a RK scheme can be described via a Butcher tableau

$$
\begin{array}{c|c}
\mathbf{c} & A \\
\hline & \mathbf{w}^{t}
\end{array} .
$$


Typical examples [9, 27] are the first-order Forward and Backward Euler (FE/BE) schemes and also the general Heun methods (including the midpoint and trapezoidal rules). Some particularly efficient implicit RK schemes are the Diagonally Implicit RK (DIRK) schemes [18 for which $A$ is a lower triangular matrix. While being implicit schemes, they can sometimes be explicitly implemented, reducing their computational cost. From a general viewpoint, explicit schemes are usually well-adapted to nonlinear problems but may suffer from severe time step restrictions. Implicit schemes are characterized by better stability properties but may be impractical due to their high computational cost for nonlinear problems.

When problem (4) involves several kinds of operators then different suitable RK discretizations may be used to integrate each of them. The resulting strategy is known as Additive RK (ARK) [27] and IMEX schemes appear as particular ARK schemes consisting in the sum of an explicit and of an implicit discretization. This technique has already been successfully applied in other contexts with important applications, as for instance in [22, 23] for kinetic equations. To implement an IMEX scheme, let us split the ODE (4) as

$$
\partial_{t} \psi=f^{\mathrm{IM}}(t, \psi)+f^{\mathrm{EX}}(t, \psi),
$$

where $f^{\mathrm{IM}}$ and $f^{\mathrm{EX}}$ are associated with the linear and nonlinear parts of the operator, respectively. Here, linear means that the involved operators can be efficiently inverted and nonlinear that the contribution is integrated in time at almost no cost. As a consequence, the global scheme has a reduced computational complexity. A compact representation of an IMEX scheme is through a double Butcher tableau, describing the implicit and explicit (with tilde) parts, given by

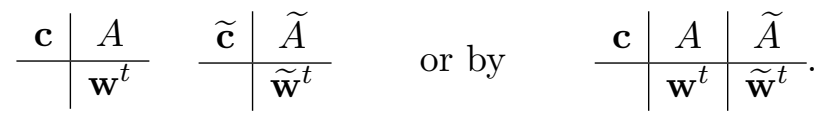

For the right tableau, the additional assumption $\widetilde{\mathbf{c}}=\mathbf{c}$, which is usually considered for high-order schemes, holds. If a DIRK tableau is used, the RK-IMEX discretization with (6) for (5) reads

$$
\begin{aligned}
& K^{m}=\psi^{n}+\Delta t \sum_{\ell=1}^{m} a_{m \ell} f^{\mathrm{IM}}\left(t^{n \ell}, K^{\ell}\right)+\Delta t \sum_{\ell=1}^{m-1} \widetilde{a}_{m \ell} f^{\mathrm{EX}}\left(t^{n \ell}, K^{\ell}\right), \quad \text { for } m=1, \ldots, \nu, \\
& \psi^{n+1}=\psi^{n}+\Delta t \sum_{\ell=1}^{\nu} w_{\ell} f^{\mathrm{IM}}\left(t^{n \ell}, K^{\ell}\right)+\Delta t \sum_{\ell=1}^{\nu} \widetilde{w}_{\ell} f^{\mathrm{EX}}\left(t^{n \ell}, K^{\ell}\right),
\end{aligned}
$$

where the second sum over $\ell$ in the first expression is not taken into account for $m=1$. The definition of $f^{\mathrm{IM}, \mathrm{EX}}$ is not a trivial task. There is no general rule to guarantee some optimal performances of the IMEX scheme. Essentially, the choice is related to the properties which are the most important for the user, e.g. to ensure the maximal stability, to get a high accuracy or to obtain an efficient computation. In some cases, even memory issues could serve as valuable guidelines.

Let us now come back to the example of the Rashba spin-orbit coupling system (1). We propose to only include the Laplace operator in the implicit part. Such a choice results from two important remarks: firstly, an explicit Laplace operator would lead to a very severe time step restriction and, secondly, the Laplace operator can be inverted in the Fourier space at almost no computational cost. Moreover, as we will see in the sequel of the paper, this approach leads to a fully explicit global scheme. Let us set, for $j=1,2$,

$$
\begin{aligned}
& f^{\mathrm{IM}}\left(t, \psi_{j}\right):=f^{\mathrm{IM}}\left(\psi_{j}\right)=\frac{i}{2} \Delta \psi_{j} \\
& f_{j}^{\mathrm{EX}}\left(t, \psi_{1}, \psi_{2}\right)=-i\left[\left(V_{j}+f_{j}\left(\psi_{1}, \psi_{2}\right)-\Omega L_{z}\right) \psi_{j}-\kappa R_{j} \psi_{3-j}-\lambda \psi_{3-j}\right] .
\end{aligned}
$$

We introduce some stages $K_{j}^{m}$ for each component, $j=1,2$. By using these notations, the semi-discrete IMEX-RK scheme for system (1) writes, for both species $j=1,2$,

$$
\left\{\begin{array}{l}
K_{j}^{m}=\psi_{j}^{n}+\Delta t \sum_{\ell=1}^{m} a_{m \ell} f^{\mathrm{IM}}\left(K_{j}^{\ell}\right)+\Delta t \sum_{\ell=1}^{m-1} \widetilde{a}_{m \ell} f_{j}^{\mathrm{EX}}\left(t^{n \ell}, K_{1}^{\ell}, K_{2}^{\ell}\right), \text { for } m=1, \ldots, \nu, \\
\psi_{j}^{n+1}=\psi_{j}^{n}+\Delta t \sum_{\ell=1}^{\nu} w_{\ell} f^{\mathrm{IM}}\left(K_{j}^{\ell}\right)+\Delta t \sum_{\ell=1}^{\nu} \widetilde{w}_{\ell} f_{j}^{\mathrm{EX}}\left(t^{n \ell}, K_{1}^{\ell}, K_{2}^{\ell}\right) .
\end{array}\right.
$$


Further possible operator decompositions into explicit and implicit parts have been tested. The proposed one, i.e. (7), leads to the best results, most particularly regarding the computational times. In particular, including any other term into the implicit part implies the solution of large linear or even nonlinear systems, e.g. through preconditioned Krylov subspace iterative solvers [5]. As a consequence, this increases the global cost of the scheme.

Let us remark that IMEX schemes do not lead to an exact evaluation at the discrete level of the macroscopic quantities. This is a drawback of the IMEX schemes for Schrödinger-type problems. Nevertheless, we will show through some numerical experiments (section 3) that the computation of macroscopic quantities is highly accurate. Mass conserving schemes are however available (see e.g. 27] for general ARK schemes and [14] for a class of fully implicit mass conserving schemes) while there is no energy conserving IMEX scheme. This situation is relatively similar to the TSSP schemes.

Finally, let us note that an alternative formulation of system (1) is to use a Lagrangian coordinates system to get rid of the rotation term. The price to pay is that a new time-dependent potential $\widetilde{V}_{j}(t, \mathbf{x})$ appears 8$]$. Without giving any detail, all the IMEXSP schemes presented here can be directly applied. More precisely, if system (1) (for $\kappa=0$ ) is written in the Lagrangian coordinates system, then it can be proven that the implicit part $f^{\mathrm{IM}}$ of the IMEX scheme remains the same while the explicit function is now

$$
f_{j}^{\mathrm{EX}}\left(t, \psi_{1}, \psi_{2}\right)=-i\left[\left(\widetilde{V}_{j}(t, \mathbf{x})+f_{j}\left(\psi_{1}, \psi_{2}\right)\right) \psi_{j}-\lambda \psi_{3-j}\right]
$$

\subsection{Adaptive time step strategy for the IMEX schemes}

Adaptive time stepping for IMEX-RK schemes can be defined by exploiting the techniques developed for RK schemes [34] and most particularly for ARK schemes [17, 27]. The main idea is to compute a second solution $\psi^{n+1}$ (eventually one for each component) at each time step with an order of accuracy different from the reference scheme and to use it to estimate an "optimal" time step $\Delta t^{n}$ to perform the iteration from $t^{n}$ to $t^{n+1}$. Standard criteria essentially force the distribution of the local error for the wave function to remain below a fixed (user-defined) tolerance at each time step. Other indicators may be used and based for instance on macroscopic quantities, i.e. the mass or the energy. Since this strategy leads to an algorithm that always controls the error of the lower order solution, it is then possible to define $\psi$ either as a higher order estimate, in which case the algorithm can be directly used, either as a lower order approximation and then an extrapolation technique is required for recovering the higher order approximation. In the present paper, we consider the second approach. The crucial point is that we compute the auxiliary solution $\psi$ at a very limited additional cost. Indeed, we use the already computed stages (which are the most time consuming for RK schemes) and simply exploit different (suitable) weights for $\underline{\psi}$. To compute $\underline{\psi}^{n+1}$, we consider a new pair of weights vectors, $\underline{\mathbf{w}}$ for the implicit and $\underline{\widetilde{\mathbf{w}}}$ for the explicit solvers, and use the same stages $K$ that have already been computed for $\psi^{n+1}$. This second scheme is usually called embedded scheme, the approximation $\psi^{n+1}$ being the associated embedded solution. The resulting full IMEX Butcher tableau including the embedded formulae reads

\begin{tabular}{c|c|c}
$\mathbf{c}$ & $A$ & $\widetilde{A}$ \\
\hline & $\mathbf{w}$ & $\widetilde{\mathbf{w}}$ \\
& $\underline{\mathbf{w}}$ & $\underline{\widetilde{\mathbf{w}}}$
\end{tabular}

and the embedded solution of system $(8)$ is given by

$$
\underline{\psi}_{j}^{n+1}=\psi_{j}^{n}+\Delta t \sum_{\ell=1}^{\nu} \underline{w}_{\ell} f^{\mathrm{IM}}\left(K_{j}^{\ell}\right)+\Delta t \sum_{\ell=1}^{\nu} \underline{\widetilde{w}}_{\ell} f_{j}^{\mathrm{EX}}\left(t^{n \ell}, K_{1}^{\ell}, K_{2}^{\ell}\right) .
$$

We denote these schemes by $\operatorname{IMEX}(q) p$, with $q$ the order of the original scheme and $p$ the order of the embedded one. We consider schemes for which $q=p+1$ but other combinations may also be possible. We implement the variable time step technique by using some local error indicators $\delta^{n}=\delta^{n}\left(\psi^{n+1}, \psi^{n+1}\right)$ at each time step but for different quantities. More precisely, we tested the $L^{2}$-norm indicator based on $\delta^{n}=\left\|\psi^{n+1}-\psi^{n+1}\right\|_{2}$, the energybased indicator $\delta^{n}=\left|E_{\psi}\left(t^{n+1}\right)-E_{\psi}\left(t^{n+1}\right)\right|$ and finally the standard $L^{\infty}$-norm indicator $\delta^{n}=\left\|\psi^{n+1}-\underline{\psi}^{n+1}\right\|_{\infty}$. From the numerical simulations, choosing one of the three quantities does not significantly change the results. 
To fix the ideas, we always use the energy-based indicator in this paper. We impose the accuracy condition $\delta^{n}=C\left(\Delta t^{n}\right)^{q} \leq \tau$, where $\tau$ is a user-defined constant representing the expected accuracy and $C>0$ is a realvalued constant related to the chosen $\operatorname{IMEX}(q) p$ scheme. Clearly, this inequality can be satisfied for a suitable choice of the (local) time step $\Delta t^{n}$ : by changing $\Delta t^{n} \mapsto \Delta t_{\text {new }}^{n}=\alpha \Delta t^{n}$, one gets $\delta^{n} \mapsto \alpha^{q} \delta^{n}$, leading to [34]

$$
\alpha \leq\left(\frac{\tau}{\delta^{n}}\right)^{\frac{1}{q}} .
$$

To avoid too many costly rejections, we accept the result of the iteration if the new time step $\Delta t_{\text {new }}^{n}$ belongs to the interval $\left[c_{m} \Delta t^{n} ; c_{M} \Delta t^{n}\right]$. The positive real-valued constant $c_{m}$ allows for a small loss of accuracy by slightly relaxing the inequality (11) for efficiency reasons. Therefore, $c_{m}$ is chosen a little bit smaller than $1\left(c_{m}=0.95\right.$ in all the simulations here). Now, if $\Delta t^{n}$ leads to an error $\delta^{n}$ which is significantly smaller than the required accuracy, then a larger local time step can be chosen. However, to avoid some spurious oscillations [34, the increase of $\Delta t^{n}$ must not be too sharp. We impose this condition by forcing $\Delta t_{\text {new }}^{n}$ to be smaller than $c_{M} \Delta t^{n}$ (in the following, we fix $\left.c_{M}=2\right)$. Like in [27], we introduce a parameter $c_{\mathrm{vts}} \leq 1$ (=0.9 in the simulations) between two successive time steps. This constant improves the accuracy but is not mandatory as we observed in our computations and could also be fixed to 1 (see e.g. [34]). For a multi-components problem, one considers the smallest time step among the resulting ones for each component. In summary, we repeat the iteration from $t^{n}$ to $t^{n+1}$ with $\Delta t_{\text {new }}^{n}$ if $\Delta t_{\text {new }}^{n}<c_{m} \Delta t^{n}$ or $\Delta t_{\text {new }}^{n}>c_{M} \Delta t^{n}$.

Finally, given two initial states $\psi_{j}^{0}$ at $t^{0}$, for $j=1,2$, and an initial time step $\Delta t^{0}$ the adaptive time step algorithm is given by

\section{while do}

compute $\psi_{j}^{n+1}$ by using (8) and $\underline{\psi}_{j}^{n+1}$ by using $(10)$, with $\Delta t=\Delta t^{n}$ and $j=1,2$;

compute the error indicator $\delta^{n}=\max _{j=1,2} \delta^{n}\left(\psi_{j}^{n+1}, \underline{\psi}_{j}^{n+1}\right)$;

compute the new time step via

$$
\Delta t_{\text {new }}^{n}=\Delta t^{n} c_{\mathrm{vts}}\left(\frac{\tau}{\delta^{n}}\right)^{\frac{1}{q}}
$$

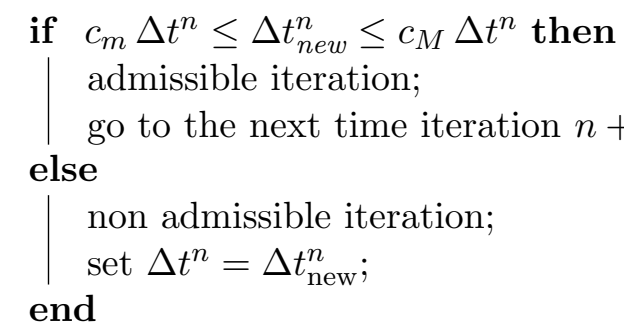

Algorithm 1: Adaptive time step algorithm to compute $\Delta t^{n}$ to get the expected accuracy from $t^{n}$ to $t^{n+1}$ (for the two-components problem);

Except for the first time iteration and if the initial time step is not correctly chosen, the above procedure leads to a negligible additional cost because of the very small number of rejections.

\subsection{Fourier pseudo-spectral discretization in space}

We now present the pseudo-spectral Fourier discretization in space [4, 6] associated with a semi-discrete IMEXRK scheme. For conciseness, we only explain the details for the two-dimensional case (i.e. $d=2$ ), the extension to the one- and three-dimensional situations being straightforward. In addition, considering other systems of NLSE/GPE is also possible and relatively direct.

Let us introduce the spatially bounded domain $\mathcal{D}=]-a_{x} ; a_{x}[\times]-a_{y} ; a_{y}[$. Since the physics related to the NLSE/GPE that we consider is located inside the computational box $\mathcal{D}$, periodic boundary conditions can be set on $\psi_{j}$ at the boundary $\partial \mathcal{D}$, for $j=1,2$. Let us remark that other boundary conditions like homogeneous Dirichlet or Neumann boundary conditions could also be imposed, resulting then in the use of sine or cosine 
transforms instead of Fourier series expansions and FFTs. Let us now introduce the discrete grid with uniformly sampled points: $\mathcal{D}_{N_{x} N_{y}}:=\left\{\mathbf{x}_{k_{1}, k_{2}}=\left(x_{k_{1}}, y_{k_{2}}\right)\right\}_{\left(k_{1}, k_{2}\right) \in \mathcal{O}_{N_{x} N_{y}}}$, where

$$
\mathcal{O}_{N_{x} N_{y}}:=\left\{\left(k_{1}, k_{2}\right) \in \mathbb{N}^{2} \text { such that } h=0, \ldots, N_{x}-1, k=0, \ldots, N_{y}-1\right\}
$$

and where $N_{x}$ and $N_{y}$ are two even positive integers. We define

$$
x_{k_{1}+1}-x_{k_{1}}=h_{x}=2 a_{x} / N_{x}, \quad y_{k_{2}+1}-y_{k_{2}}=h_{y}=2 a_{y} / N_{y} .
$$

The associated discrete Fourier frequencies are given by $\boldsymbol{\zeta}_{p q}=\left(\xi_{p}, \eta_{q}\right)$, with $\xi_{p}=p \pi / a_{x},-N_{x} / 2 \leq p \leq N_{x} / 2-1$, and $\eta_{q}=q \pi / a_{y},-N_{y} / 2 \leq q \leq N_{y} / 2-1$. We introduce the partial Fourier pseudo-spectral approximations $\widetilde{\psi}_{j}$ of the functions $\psi_{j}$ in the $x$ - and $y$-directions, $j=1,2$, as

$$
\widetilde{\psi}_{j}(t, x, y)=\frac{1}{N_{x}} \sum_{p=-N_{x} / 2}^{N_{x} / 2-1} \widehat{\widetilde{\psi}_{j, p}(t, y)} e^{i \xi_{p}\left(x+a_{x}\right)}, \quad \widetilde{\psi}_{j}(t, x, y)=\frac{1}{N_{y}} \sum_{q=-N_{y} / 2}^{N_{y} / 2-1} \widehat{\widetilde{\psi}_{j, q}}(t, x) e^{i \eta_{q}\left(y+a_{y}\right)} \text {, }
$$

where $\widehat{\widetilde{\psi}_{j, p}}$ and $\widehat{\widetilde{\psi}_{j, q}}$ respectively denote the Fourier coefficients in the $x$ - and $y$-directions

$$
\widehat{\widetilde{\psi}_{j, p}}(t, y)=\sum_{k_{1}=0}^{N_{x}-1} \widetilde{\psi}_{j, k_{1}}(t, y) e^{-i \xi_{p}\left(x_{k_{1}}+a_{x}\right)}, \quad \widehat{\widetilde{\psi}_{j, q}}(t, x)=\sum_{k_{2}=0}^{N_{y}-1} \widetilde{\psi}_{j, k_{2}}(t, x) e^{-i \eta_{q}\left(y_{k_{2}}+a_{y}\right)} .
$$

In the above equation, we use the notation $\widetilde{\psi}_{j, k_{1}}(t, y)=\widetilde{\psi}_{j}\left(t, x_{k_{1}}, y\right)$ and $\widetilde{\psi}_{j, k_{2}}(t, x)=\widetilde{\psi}_{j}\left(t, x, y_{k_{2}}\right)$. The evaluation of the different operators is made as follows. We introduce the operators $\mathbb{I}, \mathbb{V}$ and $\left[\left[f_{j}\left(\psi_{1}, \psi_{2}\right)\right]\right]$ that apply pointwise in the physical space

$$
\mathbb{I}_{k_{1}, k_{2}}:=\delta_{k_{1}, k_{2}}, \quad \mathbb{V}_{j, k_{1}, k_{2}}:=V_{j}\left(\mathbf{x}_{k_{1}, k_{2}}\right), \quad\left[\left[f_{j}\left(\boldsymbol{\psi}_{1}, \boldsymbol{\psi}_{2}\right)\right]\right]_{k_{1}, k_{2}}=f_{j}\left(\psi_{1, k_{1}, k_{2}}, \psi_{2, k_{1}, k_{2}}\right),
$$

for $\left(k_{1}, k_{2}\right) \in \mathcal{O}_{N_{x} N_{y}}$. The symbol $\delta_{k_{1}, k_{2}}$ denotes the Dirac delta symbol which is equal to 1 if and only if $k_{1}=k_{2}$ and 0 otherwise. Let us introduce the following four operators $\left[\left[\partial_{x}^{2}\right]\right],\left[\left[\partial_{y}^{2}\right]\right], y\left[\left[\partial_{x}\right]\right]$ and $x\left[\left[\partial_{y}\right]\right]$ which are applied to $\widetilde{\psi}_{j}$ that approximates the continuous function $\psi_{j}$ for $\left(k_{1}, k_{2}\right) \in \mathcal{O}_{N_{x} N_{y}}$

$$
\begin{aligned}
& \partial_{x}^{2} \psi_{j}\left(\mathbf{x}_{k_{1}, k_{2}}\right) \approx\left(\left[\left[\partial_{x}^{2}\right]\right] \widetilde{\psi}_{j}\right)_{k_{1}, k_{2}}:=-\frac{1}{N_{x}} \sum_{p=-N_{x} / 2}^{N_{x} / 2-1} \xi_{p}^{2} \widehat{\left(\widetilde{\tilde{\psi}_{j, k_{2}}}\right)} e^{i \xi_{p}\left(x_{k_{1}}+a_{x}\right)} \\
& \partial_{y}^{2} \psi_{j}\left(\mathbf{x}_{k_{1}, k_{2}}\right) \approx\left(\left[\left[\partial_{y}^{2}\right]\right] \widetilde{\psi}_{j}\right)_{k_{1}, k_{2}}:=-\frac{1}{N_{y}} \sum_{q=-N_{y} / 2}^{N_{y} / 2-1} \eta_{q}^{2} \widehat{\left(\widetilde{\tilde{\psi}_{j, k_{1}}}\right)_{q}} e^{i \eta_{q}\left(y_{k_{2}}+a_{y}\right)} \\
& \left(x \partial_{y} \psi_{j}\right)\left(\mathbf{x}_{k_{1}, k_{2}}\right) \approx\left(x\left[\left[\partial_{y}\right]\right] \widetilde{\psi}_{j}\right)_{k_{1}, k_{2}}:=\frac{1}{N_{y}} \sum_{q=-N_{y} / 2}^{N_{y} / 2-1} i x_{k_{1}} \eta_{q} \widehat{\left(\widetilde{\tilde{\psi}_{j, k_{1}}}\right)_{q}} e^{i \eta_{q}\left(y_{k_{2}}+a_{y}\right)} \\
& \left(y \partial_{x} \psi_{j}\right)\left(\mathbf{x}_{k_{1}, k_{2}}\right) \approx\left(y\left[\left[\partial_{x}\right]\right] \widetilde{\psi}_{j}\right)_{k_{1}, k_{2}}:=\frac{1}{N_{x}} \sum_{p=-N_{x} / 2}^{N_{x} / 2-1} i y_{k_{2}} \xi_{p} \widehat{\left(\widetilde{\psi}_{j, k_{2}}\right)} e_{p}^{i \xi_{p}\left(x_{k_{1}}+a_{x}\right)}
\end{aligned}
$$

We define the associated discrete operators acting from $\mathbb{C}^{M}$ to $\mathbb{C}$, with $M=N_{x} N_{y}$ (in 2D), by

$$
[[\Delta]]:=\left[\left[\partial_{x}^{2}\right]\right]+\left[\left[\partial_{y}^{2}\right]\right], \quad\left(\mathbb{L}_{z}\right):=-i\left(x\left[\left[\partial_{y}\right]\right]-y\left[\left[\partial_{x}\right]\right]\right), \quad\left(\mathbb{R}_{j}\right)=i\left[\left[\partial_{x}\right]\right]+(-1)^{3-j}\left[\left[\partial_{y}\right]\right] .
$$

The discrete operator $[[\Delta]]$ is diagonal in the Fourier space but not $\mathbb{L}_{z}$ nor $\mathbb{R}_{j}$. We set $\boldsymbol{\psi}_{j}:=\left(\psi_{j}\left(\mathbf{x}_{k_{1}, k_{2}}\right)\right)_{\left(k_{1}, k_{2}\right) \in \mathcal{O}_{N_{x} N_{y}}}$ as the discrete unknown vector in $\mathbb{C}^{M}$. For conciseness, let us remark that we do not make the distinction between an array $\boldsymbol{\psi}$ in $\mathcal{M}_{N_{x} \times N_{y}}(\mathbb{C})$ (storage according to the $2 \mathrm{D}$ spatial grid) and the corresponding reshaped vector in $\mathbb{C}^{M}$. In the above notations, $\mathcal{M}_{N_{x} \times N_{y}}(\mathbb{C})$ designates the set of $2 \mathrm{D}$ arrays with complex coefficients. 
We denote $\widetilde{\psi}_{j}$ by $\psi_{j}$ in the sequel of the paper to simplify the notations. Let us recall that

$$
\begin{aligned}
& f^{\mathrm{IM}}\left(t, \psi_{j}\right):=\frac{i}{2} \Delta \psi_{j}=f^{\mathrm{IM}}\left(\psi_{j}\right), \\
& f_{j}^{\mathrm{EX}}\left(t, \psi_{1}, \psi_{2}\right)=-i\left[\left(V_{j}+f_{j}\left(\psi_{1}, \psi_{2}\right)-\Omega L_{z}\right) \psi_{j}-\kappa R_{j} \psi_{3-j}-\lambda \psi_{3-j}\right] .
\end{aligned}
$$

From the previous discretization in space given by equations $(12)-(13)$, we introduce the discrete explicit and implicit parts of the projected operators

$$
\begin{aligned}
& {\left[\left[f^{\mathrm{IM}}\left(\boldsymbol{\psi}_{j}\right)\right]\right]:=\frac{i}{2}[[\Delta]] \boldsymbol{\psi}_{j},} \\
& {\left[\left[f_{j}^{\mathrm{EM}}\left(t^{n \ell}, \boldsymbol{\psi}_{1}, \boldsymbol{\psi}_{2}\right)\right]\right]=-i\left(\left(\left[\left[V_{j}\right]\right]+\left[\left[f_{j}\left(\boldsymbol{\psi}_{1}, \boldsymbol{\psi}_{2}\right)-\Omega\left[\left[\mathbb{L}_{z}\right]\right]\right) \boldsymbol{\psi}_{j}-\kappa\left[\left[\mathbb{R}_{j}\right]\right] \boldsymbol{\psi}_{3-j}-\lambda \boldsymbol{\psi}_{3-j}\right) .\right.\right.}
\end{aligned}
$$

The associated discrete IMEX pseudo-SPectral (IMEXSP) scheme is then

$$
\left\{\begin{array}{l}
\mathbf{K}_{j}^{m}=\boldsymbol{\psi}_{j}^{n}+\Delta t \sum_{\ell=1}^{m} a_{m \ell}\left[\left[f^{\mathrm{IM}}\left(\mathbf{K}_{j}^{\ell}\right)\right]\right]+\Delta t \sum_{\ell=1}^{m-1} \widetilde{a}_{m \ell}\left[\left[f_{j}^{\mathrm{EM}}\left(t^{n \ell}, \mathbf{K}_{1}^{\ell}, \mathbf{K}_{2}^{\ell}\right)\right]\right], \text { for } m=1, \ldots, \nu, \\
\boldsymbol{\psi}_{j}^{n+1}=\boldsymbol{\psi}_{j}^{n}+\Delta t \sum_{\ell=1}^{\nu} w_{\ell}\left[\left[f^{\mathrm{IM}}\left(\mathbf{K}_{j}^{\ell}\right)\right]\right]+\Delta t \sum_{\ell=1}^{\nu} \widetilde{w}_{\ell}\left[\left[f_{j}^{\mathrm{EM}}\left(t^{n \ell}, \mathbf{K}_{1}^{\ell}, \mathbf{K}_{2}^{\ell}\right)\right]\right] .
\end{array}\right.
$$

If one considers now the first equation of system (14), then one gets, for $j=1,2$,

$$
\left(\mathbb{I}-\Delta t a_{m m} \frac{i}{2}[[\Delta]]\right) \mathbf{K}_{j}^{m}=\mathbf{b}_{j}^{m-1} \text { for } m=1, \ldots, \nu,
$$

setting the right-hand side as

$$
\mathbf{b}_{j}^{m-1}:=\boldsymbol{\psi}_{j}^{n}+\Delta t\left[\left[f^{\mathrm{IM}}\left(\sum_{\ell=1}^{m-1} a_{m \ell} \mathbf{K}_{j}^{\ell}\right)\right]\right]+\Delta t \sum_{\ell=1}^{m-1} \widetilde{a}_{m \ell}\left[\left[f_{j}^{\mathrm{EX}}\left(t^{n \ell}, \mathbf{K}_{1}^{\ell}, \mathbf{K}_{2}^{\ell}\right)\right]\right],
$$

by using the linearity of $\left[\left[f^{\mathrm{IM}}\right]\right]$. However, since the operator that defines $[15$ can be directly inverted in the Fourier space (this is a constant coefficients perturbed Laplacian operator and therefore the corresponding matrix is diagonal in the Fourier space), the unknown $\mathbf{K}_{j}^{m}$ is explicitly computed (thanks to the FFT and iFFT functions) by

$$
\mathbf{K}_{j}^{m}=\left(\mathbb{I}-\Delta t a_{m m} \frac{i}{2}[[\Delta]]\right)^{-1} \mathbf{b}_{j}^{m-1} \text { for } m=1, \ldots, \nu,
$$

the unknown $\boldsymbol{\psi}_{j}^{n+1}$ being next obtained through the second equation of 14. As a consequence, an IMEXSP scheme of order $q$ (that we denote by $\operatorname{IMEXSP}(q)$ ) can be considered as a fully explicit scheme. The global cost is $\mathcal{O}(M \log M)$ and increases linearly with respect to the number of stages $\nu$. If the Lagrangian coordinates system is used, then one has to adapt the scheme to the definition of the explicit part given by Eq. (9).

\subsection{Time-splitting discretizations}

Time-Splitting (TS) schemes are often used for solving NLSE/GPE [6, 10, 11, 15, 20]. Even if TS schemes are accurate and efficient, they may be limited in some circumstances, most particularly if one wants to build high-order time discretizations. Let us consider an equation of the form

$$
\partial_{t} \psi(t, \mathbf{x})=A \psi(t, \mathbf{x})+B \psi(t, \mathbf{x}),
$$

with an initial data $\psi(0, \mathbf{x})=\psi_{0}(\mathbf{x})$ and two operators $A$ and $B$. Then, the solution writes: $\psi(t, \mathbf{x})=$ $e^{(A+B) t} \psi_{0}(\mathbf{x})$. The TS approach basically consists in computing an approximation of the solution through a combination of $e^{A t} \psi_{0}$ and $e^{B t} \psi_{0}$, solutions to $\partial_{t} \psi(t, \mathbf{x})=A \psi(t, \mathbf{x})$ and $\partial_{t} \psi(t, \mathbf{x})=B \psi(t, \mathbf{x})$, respectively. The approximate solution is then of the form

$$
\psi(t+\Delta t, \mathbf{x}) \approx e^{a_{1} A \Delta t} e^{b_{1} B \Delta t} \cdot \ldots \cdot e^{a_{p} A \Delta t} e^{b_{p} B \Delta t} \psi(t, \mathbf{x}),
$$


where $a_{k}, b_{k} \in \mathbb{R}$, for $k=1, \ldots, p$, are some suitable weights designed to ensure the order of accuracy. For example, the first-order Lie splitting scheme considers $a_{1}=b_{1}=1$ and the second-order Strang splitting scheme takes $a_{1}=a_{2}=1 / 2$ and $b_{1}=1$. A fourth-order TS scheme [36] is given by: $a_{1}=a_{4}=\delta^{-1} / 2, a_{2}=a_{2}=1 / 2-a_{2}$, $b_{1}=b_{3}=\delta^{-1}, b_{2}=1-2 b_{1}, b_{4}=0$, with $\delta=2-2^{1 / 3}$. A sixth-order TS scheme is based on the coefficients derived in [16]. In section 3, these TS schemes (of orders 2, 4 and 6) are used as references for a numerical comparison with the IMEX-RK schemes (both using Fourier pseudo-spectral discretizations in space).

A standard way to apply the TS scheme to a one-component GPE with rotation term is to use the decomposition $A=i\left(\Delta / 2+\Omega L_{z}\right)$ and $B=-i(V+f(\psi))$. The equation related to $A$ leads to the solution of a linear system at each time step. For $B$, the terms can be sometimes analytically integrated. This is for example the case when considering the standard GPE with quadratic potential and cubic nonlinearity but without rotation [6, 10] or in Lagrangian coordinates when a rotating term is included [11, 12]. This results in very efficient TS schemes. Similar situations can be met in [31] for multi-components.

However, when the potential $V$ is time-dependent, in many cases an exact integration of $e^{-i \int_{t}^{t+\Delta t} V(\tau, \mathbf{x}) d \tau}$ is not possible either in the Lagrangian or initial settings and a numerical integration is required. Here, two main problems arise. The first one is that for TS schemes of order higher than one, integrals at proper times need to be computed to get the expected order. For instance, in the RK context, the correct values are given by the coefficients $\mathbf{c}$ in (6). Several solutions are available in the literature [15, 20, 33, for particular cases but a general theory still lacks. The second more practical issue is that for high-order TS schemes quadrature rules (of at least the same order) are then needed and thus their global computational costs dramatically increase. These problems appear whenever the "Hamiltonian" is time-dependent and in this case designing high-order TS schemes is a nontrivial task which involves techniques for non-autonomous equations.

In addition, if the rotating term is included in the operator $A$, a possible alternative to solving the linear system is to use Alternating Direction Implicit (ADI) techniques [12. However, this approach has a priori some limitations since the splitting arising in the ADI method is only second-order accurate in space, limiting therefore the global accuracy of the scheme. This point is independent of the time discretization. A similar difficulty arises for the Rashba spin-orbit coupling due to first-order partial derivatives in space. Typical situations in which system (1) cannot be simplified by using the Lagrangian technique are e.g. the multi-components case if the rotation speeds are different for the two species (i.e. the equations include the terms $\Omega_{1} L_{z}$ and $\Omega_{2} L_{z}$, with $\Omega_{1} \neq \Omega_{2}$ ), if a Rashba spin-orbit-like coupling is present and more generally for all the cases where there is an asymmetry which cannot be simplified.

When coupled with a Fourier pseudo-spectral discretization in space, the resulting scheme is called TSSP. They are denoted by $\operatorname{TSSP}(q)$ to precise the order $q$ of accuracy in time $(q=2,4,6)$. Let us finally recall that adaptive time step techniques have also been developed for TSSP schemes [21].

\section{Numerical results}

We present now a thorough numerical study of the IMEXSP schemes of various orders and a comparison with the TSSP schemes. One important goal of this numerical study is to analyze which IMEX schemes perform best, most particularly in comparison with some references TSSP schemes that can be found in the literature. We show that high-order IMEXSP schemes generally achieve very good results. In particular, they are efficient and robust when the adaptive time stepping strategy is used for computing the wave function and the associated macroscopic quantities.

To evaluate the accuracy of the methods, we compare the evolution of the three following quantities

- the relative error $e_{\mathrm{S}}^{n}$ between the numerical $\psi^{n}$ and the exact $\psi_{\mathrm{ex}}=\psi_{\mathrm{ex}}(t, \cdot)$ solutions at time $t_{n}$

$$
e_{\mathrm{S}}^{n}=\frac{\left\|\psi^{n}(\cdot)-\psi_{\mathrm{ex}}\left(t^{n}, \cdot\right)\right\|_{2}}{\left\|\psi_{\mathrm{ex}}\left(t^{n}, \cdot\right)\right\|_{2}}
$$

- the relative mass error

$$
e_{N}^{n}=\left|\frac{N_{\psi^{n}}-N_{\psi_{\mathrm{ex}}}\left(t_{n}\right)}{N_{\psi_{\mathrm{ex}}}\left(t_{n}\right)}\right|
$$


- and the relative energy error

$$
e_{E}^{n}=\left|\frac{E_{\psi^{n}}-E_{\psi_{\mathrm{ex}}}\left(t_{n}\right)}{E_{\psi_{\mathrm{ex}}}\left(t_{n}\right)}\right| .
$$

For some of the examples considered here, the mass or/and the energy are conserved at the continuous level, i.e. for any $n \in \mathbb{N}, N_{\psi_{\mathrm{ex}}}\left(t_{n}\right)=N_{\psi_{0}}$ and $E_{\psi_{\mathrm{ex}}}\left(t_{n}\right)=E_{\psi_{0}}$, respectively. We are mainly interested in the maximal errors $e_{\mathrm{S}, N, E}^{\infty}$ of these quantities over the global time interval $\left[0 ; T_{\mathrm{f}}\right]$ with respect to the time step $\Delta t$. The quantity $e_{\mathrm{S}, N, E}^{\infty}$ are defined by $e_{\mathrm{S}, N, E}^{\infty}:=\max _{0 \leq n \leq N_{\mathrm{f}}} e_{\mathrm{S}, N, E}^{n}$, where the integer $N_{\mathrm{f}}$ is the index corresponding to the last iteration at final time $T_{\mathrm{f}}$. Moreover, when available, we also report the computational times for both the IMEXSP and TSSP schemes to achieve a given accuracy on the wave function, the mass and the energy.

Let us now come to the IMEX time-integrator. We refer to [17, 27] and references therein for general reviews on all these aspects. The coefficients of RK or ARK schemes must satisfy some conditions to guarantee when possible suitable properties like consistency, stability (the latter called A-stability for stiff ODEs), a given order of accuracy, an exact computation of some macroscopic quantities (i.e. linear or quadratic integrals) or other quantities depending on the problem under study. All the selected Butcher tableaux for the IMEX methods used here are A-stable. When it is possible, we consider Implicitly Stiffly Accurate (ISA) or Globally Stiffly Accurate (GSA) schemes: the former class is given by the property $\mathbf{w}^{t}=A_{\nu}$ (the last row $A_{\nu}$ of $A$ is equal to the weights vector $\mathbf{w}$ ) and the latter is a subset of the ISA methods with a similar condition on the explicit part, $\widetilde{\mathbf{w}}^{t}=\widetilde{A}_{\nu}$. The ISA property ensures good stability properties for stiff implicit terms that become important for our problem when using fine space discretizations. The GSA property implies that $\widehat{\psi}^{n+1}=\widehat{K}^{\nu}$. The numerical tests performed for the IMEXSP schemes show that the theoretical orders of convergence are always attained when suitable tableaux are used. In the sequel, we consider the following tableaux

Order 2: second-order IMEX version of the midpoint rule, the same as the Implicit-Explicit midpoint $(1,2,2)$ scheme in [9].

Order 4: the fourth-order ARK4(3)6L[2]SA scheme from [27] fails to reach a high-order of accuracy as expected. Indeed, it suffers from the order reduction phenomena when used for stiff problems. Therefore, we consider three fourth-order GSA schemes from [28]: the 7 stages scheme ARK.4.A.2 and the two 6 stages ARK.4.A.3.2 and ARK.4.A.4.2 schemes. Among these, the best results are obtained with ARK.4.A.4.2 which provides in general a similar accuracy as the two others but at a lower computational cost (since it uses less stages and has a lower convergence time). In the following, the fourth-order tableau ARK.4.A.4.2 is the one considered by default (see also the end of subsection 3.3.2 for ARK.4.A.2).

Order 5: the fifth-order and 8 stages ARK5(4)8L[2]SA scheme in [27].

Other schemes of orders 1 and 3 have also been implemented but we do not report the results here.

Starting from the default ARK.4.A.4.2 IMEX tableau, we define a new embedded formula (denoted by IMEXSP(4)3) to implement the adaptive time step algorithm: the weights for the computation of the embedded solution are given by

$$
\underline{\mathbf{w}}=\mathbf{w} \quad \text { and } \quad \underline{\widetilde{\mathbf{w}}}=\left(\frac{1}{6}, 0, \frac{2}{3}, \frac{1}{12}, \frac{1}{12}, 0\right) .
$$

The choice of these coefficients yields a third-order approximation that can be used in formula (10). In the present paper, we exploit the variable time step technique by using the proposed fourth-order or the fifth-order IMEXSP scheme, which already has a fourth-order embedded formula [27. We denote by $\operatorname{IMEXSP}(q)$ the IMEX scheme with a tableau of order $q(q=2,4,5$ are shown in the simulations). As already said, we designate by $\operatorname{IMEXSP}(q) p$ the corresponding variable time stepping scheme. For some examples, we perform a numerical study to compare the fixed and adaptive time stepping techniques. In particular, we show how the parameter $\tau$ in (11) influences the results since small values of $\tau$ increase the accuracy. For a given initial time step, the algorithm automatically chooses the time step which gives the accuracy forced by the parameter $\tau$. To obtain some highly accurate results, it is usually necessary to fix $\tau$ to small values, i.e. $10^{-6}, \ldots, 10^{-12}$. However, the computational cost of the procedure increases for lower values of $\tau$. Finally, in some cases, memory issues may prevent the possibility to run a simulation. In this case, the low storage RK schemes may become useful. They are defined so that only a small number of stages must be stored, even for a high resolution, and the memory 
requirement is drastically reduced compared to standard RK schemes. We refer to [19 for a general review where examples of high-order low storage IMEX tableaux are given. We tested the third- and fourth-order low storage tableaux and the results were always comparable to the corresponding ones for the full tableaux. We compare the IMEXSP and TSSP schemes of different orders in an autonomous case (i.e. the cubic nonlinear Schrödinger equation) while in the two other cases we concentrate on the IMEXSP schemes to show how they behave as general solvers for NLSE/GPE.

\subsection{Example 1: the one-dimensional cubic NLSE}

Let us begin by considering a simple one-dimensional cubic NLSE

$$
i \partial_{t} \psi=-\partial_{x}^{2} \psi+\beta|\psi|^{2} \psi
$$

In this case, an exact solution exists and is given by the soliton formula [29]

$$
\psi_{\mathrm{ex}}(t, x)=\frac{2 a}{\beta} \operatorname{sech}\left[\sqrt{a}\left(\left(x-x_{0}\right)-c t\right)\right] \exp \left[i\left(\frac{c}{2}\left(x-x_{0}\right)-\left(\frac{c^{2}}{4}-a\right) t\right)\right] .
$$

The initial data is fixed to: $\psi_{0}(x)=\psi_{\mathrm{ex}}(t=0, x)$ and the final time of computation is $T_{\mathrm{f}}=5$. The parameters of the reference solution are

$$
\beta=-8, a=\frac{\beta^{2}}{16}, c=0.5, x_{0}=-2.5 .
$$

In addition, the computational domain is $x \in \mathcal{D}:=]-20,20$ [ (i.e. $a_{x}=20$ ), with $N_{x}=2^{9}$ points for the Fourier approximation. For this example, the energy $E_{\psi}$ is conserved and is defined by

$$
E_{\psi}(t)=\int_{\mathbb{R}}|\nabla \psi|^{2}+\frac{1}{2} \beta|\psi|^{4} d \mathbf{x} .
$$

We performed this (and only this) test by using the quadruple precision (i.e. 16 bytes) in order to check the convergence orders of the schemes. In Figure 1, we compare the evolution of the errors $e_{\mathrm{S}, N, E}^{\infty}$ with respect to various steps $\Delta t$ and for the IMEXSP and TSSP schemes of different orders. In the top left plot, where the error on the solution $e_{\mathrm{S}}^{\infty}$ is shown, we see that the two schemes achieve the correct convergence rates. One also observes a stagnation of the error at a much worse level than the expected accuracy in quadruple precision. The reason is that the physical truncation boundary conditions are not correctly approximated by the periodic boundary conditions when using an FFT-based discretization. One solution may be to consider larger computational domains but at the price of involving more discretization points, or to use a sine transform (based on homogeneous Dirichlet boundary conditions). The top right figure concerning the mass accuracy $e_{N}^{\infty}$ clearly shows that the TSSP scheme is exact and that the IMEXSP(4) scheme behaves like a fifth-order scheme. One may be surprised by the fact that the error of the TSSP scheme grows with smaller $\Delta t$ (see also the bottom left figure concerning the energy error $e_{E}^{\infty}$ for $\operatorname{TSSP}(4)$ and $\operatorname{TSSP}(6)$ ). This is due to the accumulation of round-off errors during the computations. This can be solved by using the strategy described in section VIII.5 in reference [25].

For this test-case, the TSSP schemes globally behave better than the IMEXSP schemes, in particular concerning the error related to the mass. Nevertheless, these results also show that the IMEXSP schemes yield some highly accurate solutions even if they are not exactly mass nor energy preserving.

\subsection{Example 2: a NLSE with time-dependent potential and cubic-plus-quintic nonlinear- ity}

The second example considers the following one-dimensional NLSE (see e.g. [13])

$$
i \partial_{t} \psi=-\partial_{x}^{2} \psi+V \psi+G_{1}|\psi|^{2} \psi+G_{2}|\psi|^{4} \psi,
$$



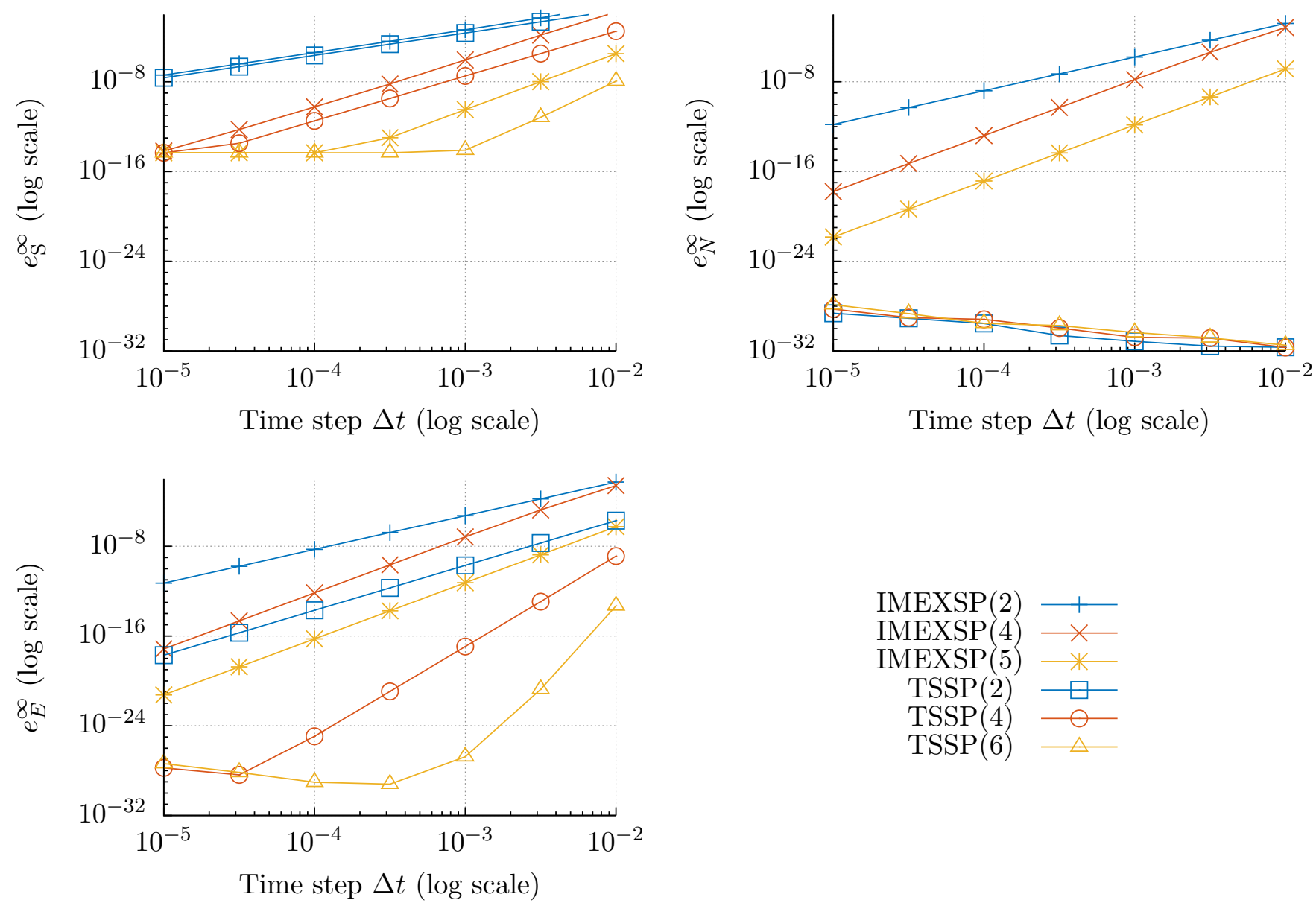

Figure 1: Example 1: evolution of the errors $e_{\mathrm{S}}^{\infty}$ (top left), $e_{N}^{\infty}$ (top right) and $e_{E}^{\infty}$ (bottom left) for the IMEXSP and TSSP schemes of different orders with respect to the uniform time step $\Delta t$.

with a cubic-plus-quintic nonlinearity and an external time-dependent potential given by $V(t, x)=\frac{x}{2} \omega^{2} \cos (\omega t)$. In this case, an exact solution is known as the bright soliton and is given by the expression

$$
\psi_{\mathrm{ex}}(t, x)=\eta \frac{\exp \left(i\left(-\frac{\omega}{2} x \sin \left(\omega t+\beta_{0}\right)-\frac{\omega^{2}}{8} t+\frac{\omega}{16} \sin \left(2 \omega t+2 \beta_{0}\right)-E_{\mathrm{c}} t\right)\right)}{\left(\sqrt{1-b} \cosh \left(2 \sqrt{-E_{\mathrm{c}}}(x-\cos (\omega t))\right)+1\right)^{1 / 2}} .
$$

The initial data is fixed to: $\psi_{0}(x)=\psi_{\mathrm{ex}}(t=0, x)$. We report the resulting computations up to final time $T_{\mathrm{f}}=5$ but also for a long time simulation up to $T_{\mathrm{f}}=100$. The parameters of the reference solution are fixed to

$$
G_{1}=-2, G_{2}=\frac{1}{2}, \omega=2, E_{\mathrm{c}}=-1, \beta_{0}=0, \eta=\sqrt{\frac{4 E_{\mathrm{c}}}{G_{1}}}, b=-16 \frac{E_{\mathrm{c}} G_{2}}{3 G_{1}^{2}} .
$$

The spatial computational domain is $\mathcal{D}:=]-80,80$ [ (i.e. $a_{x}=80$ ), with $N_{x}=2^{9}$ points for the Fourier approximation. For this example, the energy is not conserved but, since the exact solution is available, we can report the evolution of the maximum of the relative error on the energy $e_{E}^{\infty}$. In this case, we do not compare with the results of the TSSP scheme since we are solving a non-autonomous problem.

In Figure 2, we compare the evolution of the errors $e_{\mathrm{S}, N, E}^{\infty}$ with respect to $\Delta t$ for the IMEXSP schemes of different orders up to the final time $T_{\mathrm{f}}=5$. We can see that very accurate results are achieved showing that IMEXSP schemes are very well-suited for non-autonomous problems. We observe that the macroscopic quantities are very well approximated also in this case. Both the solution and the mass are accurately represented (by high-order versions) and the energy is well estimated even if there is no energy conservation. 

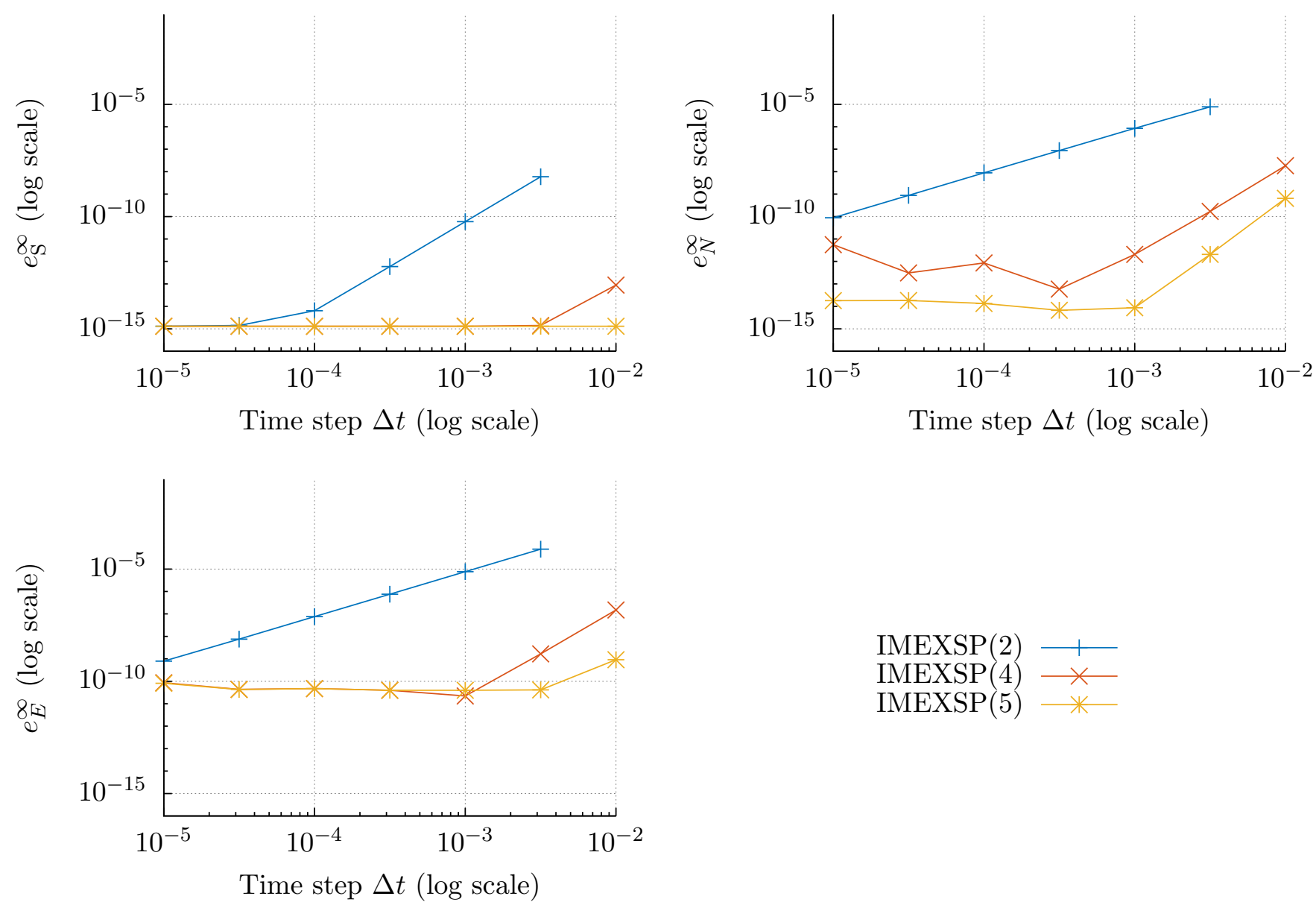

Figure 2: Example 2: evolution of the errors $e_{\mathrm{S}}^{\infty}$ (top left), $e_{N}^{\infty}$ (top right) and $e_{E}^{\infty}$ (bottom left) for the IMEXSP schemes of different orders with respect to the uniform time step $\Delta t$.

We report in Figure 3 the computational time to achieve a given a priori fixed error $e_{\mathrm{S}, N, E}^{\infty}$ for the IMEXSP schemes. We can see that a high precision is obtained for both the solution $e_{\mathrm{S}}^{\infty}$ and the mass $e_{N}^{\infty}$ while there is a slight loss of accuracy regarding the energy.

We now analyze the adaptive time step strategy introduced in section 2.2. To this end, we compare the results of the fixed and variable time step techniques. The fifth-order $\operatorname{IMEXSP}(5)$ scheme with $\Delta t=10^{-3}$ is considered for comparison since it yields the best results in terms of both accuracy and computational cost. For the adaptive time stepping strategy, we choose the $\operatorname{IMEXSP}(5) 4$ scheme with small values of $\tau$, i.e. $\tau=10^{-10}$ or $10^{-11}$, to ensure a high accuracy. We also use different starting time steps $\Delta t=10^{-2}, 10^{-3}$ and $10^{-4}$ in order to understand how this choice influences the final result. We report the evolution of the errors $e_{\mathrm{S}, N, E}^{\infty}$ on Figure 4 respectively on top left, top right and bottom left. Only curves relative to $\tau=10^{-11}$ are reported for the IMEXSP(5) 4 scheme since those for $\tau=10^{-10}$ are similar.

We can see that all the three quantities are very well described by the adaptive time step technique. Concerning the mass, we observe a slightly lower precision for the variable time step technique compared to the fixed one while both methods achieve the same accuracy on the solution and the energy. Moreover, we remark that the chosen initial time step has no effect on the results since the adaptive algorithm automatically computes the correct step. Still in Figure 4 (bottom right), we report the evolution of the time steps used by the adaptive scheme for $\tau=10^{-10}, 10^{-11}$, up to the final time $T_{\mathrm{f}}=5$. We see that the algorithm automatically chooses the time step which gives the imposed accuracy (determined by $\tau$ ). In this case, we observe that the selected time step is very close to the one used for the fixed strategy which gave the optimal results. From the number of iterations and the values of the time steps involved in the adaptive scheme, one can see that the computational cost of $\operatorname{IMEXSP}(5) 4$ is a third of that required for $\operatorname{IMEXSP}(5)$ with $\tau=10^{-10}$ and a half for $\tau=10^{-11}$. Similar 

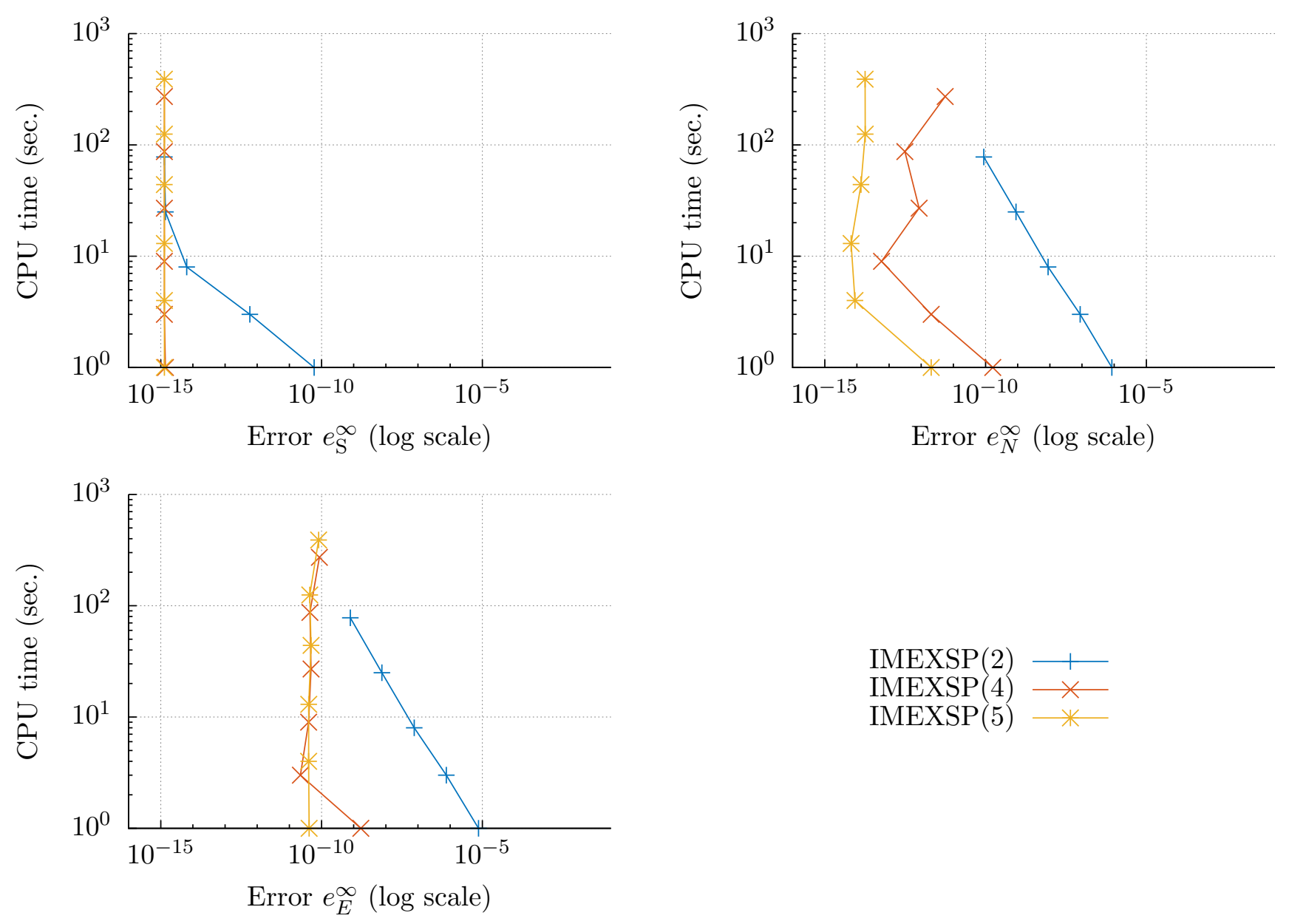

Figure 3: Example 2: CPU times (in seconds) to achieve a given accuracy for the IMEXSP schemes of various orders (the time steps $\Delta t$ are adapted to get the curves).

conclusions are obtained comparing IMEXSP(4) and IMEXSP(4)3. Thus, using the adaptive time stepping method accelerates the global computations while maintaining the a priori selected accuracy.

Let us now consider the long time behavior of the various schemes by performing the same test as before but for $T_{\mathrm{f}}=100$. We again compare the fixed and variable time step strategies. We draw on Figure 5 the evolution of the errors $e_{\mathrm{S}}^{\infty}$ (top left), $e_{N}^{\infty}$ (top right) and $e_{E}^{\infty}$ (bottom left). We report the results for the IMEXSP(5) scheme with two constant time steps $\Delta t\left(=10^{-2}, 10^{-3}\right)$ and for the $\operatorname{IMEXSP}(5) 4$ scheme for two values of the parameter $\tau\left(=10^{-10}, 10^{-11}\right)$. We see that $\operatorname{IMEXSP}(5)$ with $\Delta t=10^{-2}$ globally gives some very good results even if there is a loss of accuracy at some point. The variable time step technique achieves the same accuracy as IMEXSP(5) with $\Delta t=10^{-3}$ (i.e. the best results) concerning the solution and the energy while the latter is more accurate for the mass, as previously. The computational cost of $\operatorname{IMEXSP}(5) 4$ with $\tau=10^{-11}$ is less than a half (it ends in approximately $4.4 \times 10^{4}$ iterations) of $\operatorname{IMEXSP}(5)$. The cost of $\operatorname{IMEXSP}(5) 4$ with $\tau=10^{-10}$ is almost a quarter (ending in about $2.7 \times 10^{4}$ iterations) of the cost required for $\operatorname{IMEXSP}(5)$. A similar gain in terms of computational effort is observed when considering $\operatorname{IMEXSP}(4) 3$ vs. IMEXSP(4). The evolution of the time step is shown on Figure 5 (bottom right) for the two values of the parameter $\tau\left(=10^{-10}, 10^{-11}\right)$.

\subsection{Example 3: the two-components Rashba spin-orbit coupled system (1)}

We consider now the full model of the two-components Rashba spin-orbit coupled system (1) without (respectively in subsection 3.3.1) or with (resp. subsection 3.3.2) a rotating term. In this case, deriving a high-order TSSP scheme is nontrivial. We use the IMEXSP schemes presented in subsection 2.1 in the original fixed coordinates system. Moreover, this situation is also interesting since using the Lagrangian coordinates does not avoid the evaluation of the gradient terms. In all the simulations, we properly choose some values of the parameters 

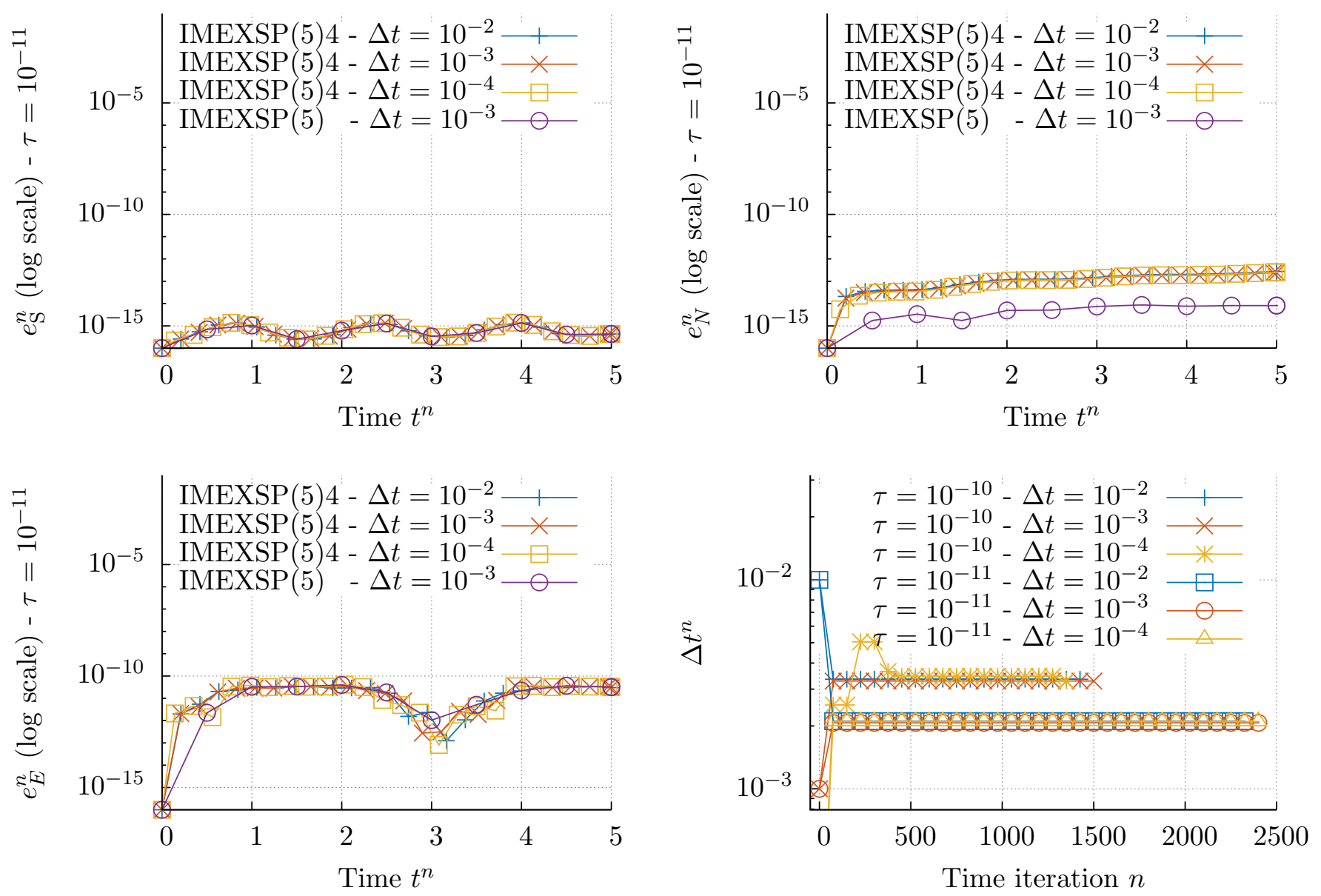

Figure 4: Example 2: evolution of the errors $e_{\mathrm{S}}^{n}$ (top left), $e_{N}^{n}$ (top right) and $e_{E}^{n}$ (bottom) for IMEXSP(5)4, with $\tau=10^{-11}$ and different initial values of $\Delta t\left(=10^{-2}, 10^{-3}, 10^{-4}\right)$, and for $\operatorname{IMEXSP}(5)$, with $\Delta t=10^{-3}$.

that correspond to some interesting physical configurations reported in [1].

\subsubsection{The non-rotating case}

Let us first consider the non-rotating spin-orbit BEC, i.e. we fix $\Omega=0$. The external potential is the harmonic well defined by (2), with $\gamma_{j, x}=1.1$ and $\gamma_{j, y}=0.9$, for $j=1,2$. The nonlinear coefficients appearing in the cubic nonlinearity (3) are $\beta_{11}=\beta_{22}=400$ and $\beta_{12}=\beta_{21}=800$. The Rashba spin-orbit interaction strength is taken as $\kappa=2$. The computational domain is $\mathcal{D}=]-10,10\left[^{2}\right.$, with $N_{x}=N_{y}=2^{9}$ points for the Fourier approximation. The final time is $T_{\mathrm{f}}=10$. The initial data is computed by GPELab ${ }^{1}$ [4, 6] as a stationary state of the same system but with $\gamma_{12, x y}=1$ and $\beta_{12}=\beta_{21}=600$.

In Figure 6, we plot the density functions $\left|\psi_{1}\right|^{2}$ (top) and $\left|\psi_{2}\right|^{2}$ (bottom) of the two components at the initial $(t=0$, left $)$ and final $\left(T_{\mathrm{f}}=10\right.$, right $)$ times obtained by using $\operatorname{IMEXSP}(5)$, with $\Delta t=10^{-3}$.

We draw in Figure 7 the time evolution of the errors on the mass $e_{N}^{n}$ (left) and the energy $e_{E}^{n}$ (right) obtained by using $\operatorname{IMEXSP}(4)$ and $\operatorname{IMEXSP}(5)$, with $\Delta t=10^{-2.5}$ and $\Delta t=10^{-3}$. We observe that these two macroscopic quantities are well-preserved.

Finally, in Table 1 we report the computational times obtained on a Dell machine with Intel core i7-3740QM CPU @ $2.70 \mathrm{GHz}$ and with 16 GB memory. We can see that the IMEXSP(4) scheme provides a good compromise between the computational time and the accuracy.

\footnotetext{
${ }^{1}$ http://gpelab.math.cnrs.fr
} 

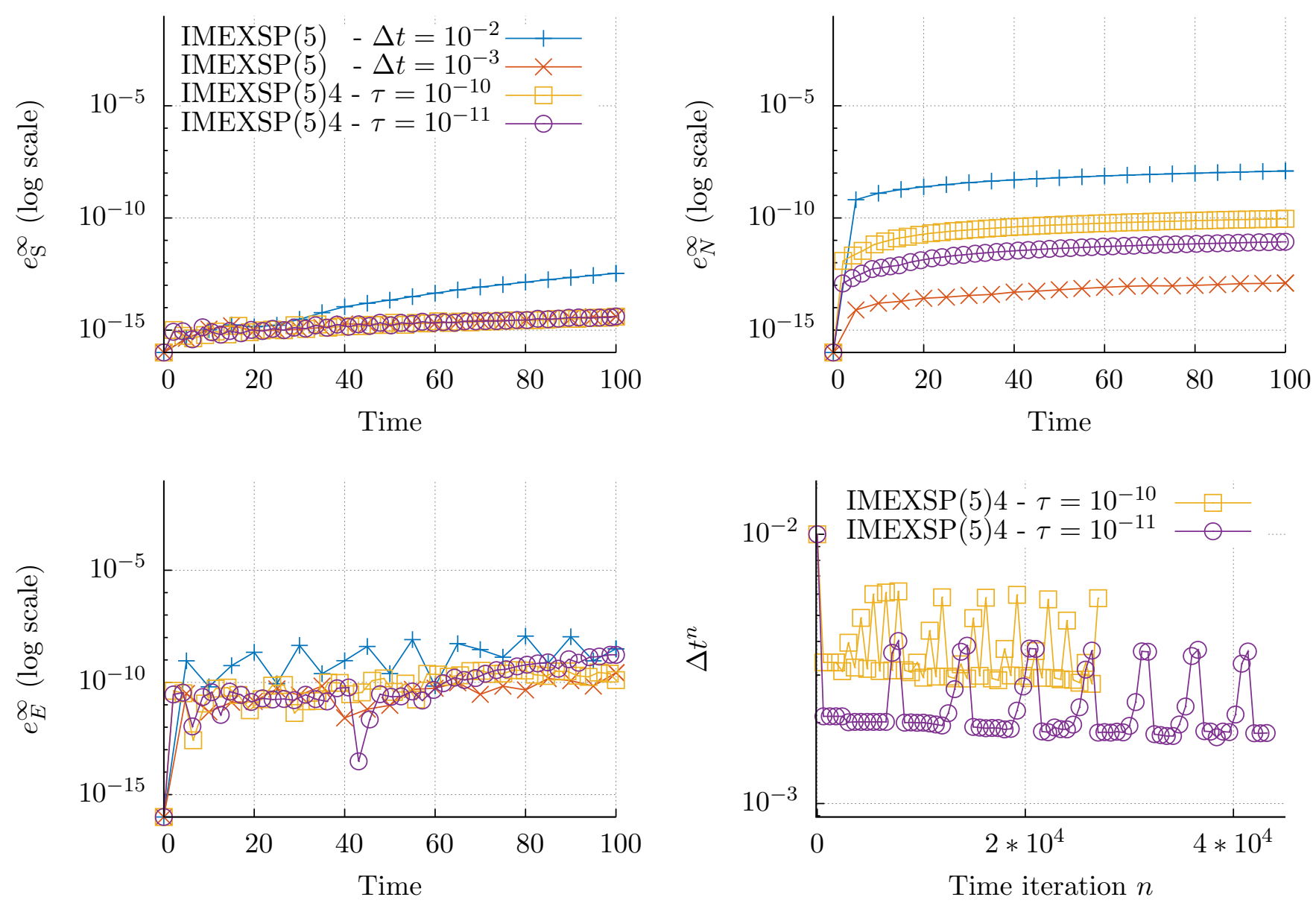

Figure 5: Example 2: long time evolution of the errors $e_{\mathrm{S}}^{\infty}$ (top left), $e_{N}^{\infty}$ (top right) and $e_{E}^{\infty}$ (bottom) for the $\operatorname{IMEXSP}(5)$ and IMEXSP(5)4 schemes.

\begin{tabular}{|c|c|c|}
\hline$\Delta t$ & IMEXSP(4) & IMEXSP(5) \\
\hline \hline $10^{-2.5}$ & $22 \mathrm{~m}$ & $40 \mathrm{~m}$ \\
$10^{-3}$ & $1 \mathrm{~h} 9 \mathrm{~m}$ & $2 \mathrm{~h} 5 \mathrm{~m}$ \\
\hline
\end{tabular}

Table 1: Example 3 (with $\Omega=0$ ): CPU times for $\operatorname{IMEXSP}(4)$ and $\operatorname{IMEXSP}(5)$ for different time steps.

\subsubsection{The rotating case}

We consider now the case of a two-components Rashba spin-orbit coupled system with a rotating term. The external potential is the quadratic potential 2 with parameters $\gamma_{j, x}=1.1$ and $\gamma_{j, y}=0.9$, for $j=1,2$. The nonlinearity strengths in (3) are fixed to $\beta_{11}=\beta_{22}=400$ and $\beta_{12}=\beta_{21}=800$ while the coupling parameter is $\kappa=2$. The rotating velocity is now $\Omega=0.4$. The computational domain $\mathcal{D}=]-10,10\left[{ }^{2}\right.$ is discretized with $N_{x}=N_{y}=2^{9}$ points for the Fourier pseudo-spectral approximation. The final time of computation is $T_{\mathrm{f}}=10$. The initial data is obtained thanks to GPELab for the same parameters but with the modifications $\gamma_{12, x y}=1$, $\Omega=0.1$ and $\kappa=1.75$.

In Figure 8, we plot the density functions $\left|\psi_{1}\right|^{2}$ (top) and $\left|\psi_{2}\right|^{2}$ (bottom) of the two components at times $t=0,2, T_{\mathrm{f}}(=10)$ by using $\operatorname{IMEXSP}(5) 4$ with $\tau=10^{-6}$. The results obtained with all the other schemes reported below are (visually) the same.

We then present and compare results obtained with the fourth- and fifth-order schemes, with and without variable time stepping strategies. In Figure 9, we report the evolution of the errors on the mass $e_{N}^{n}$ (left) and energy $e_{E}^{n}$ (right) computed by the $\operatorname{IMEXSP}(4)$ scheme with $\Delta t=10^{-3.5}$ and $\Delta t=10^{-4}$, and by $\operatorname{IMEXSP}(4) 3$ 

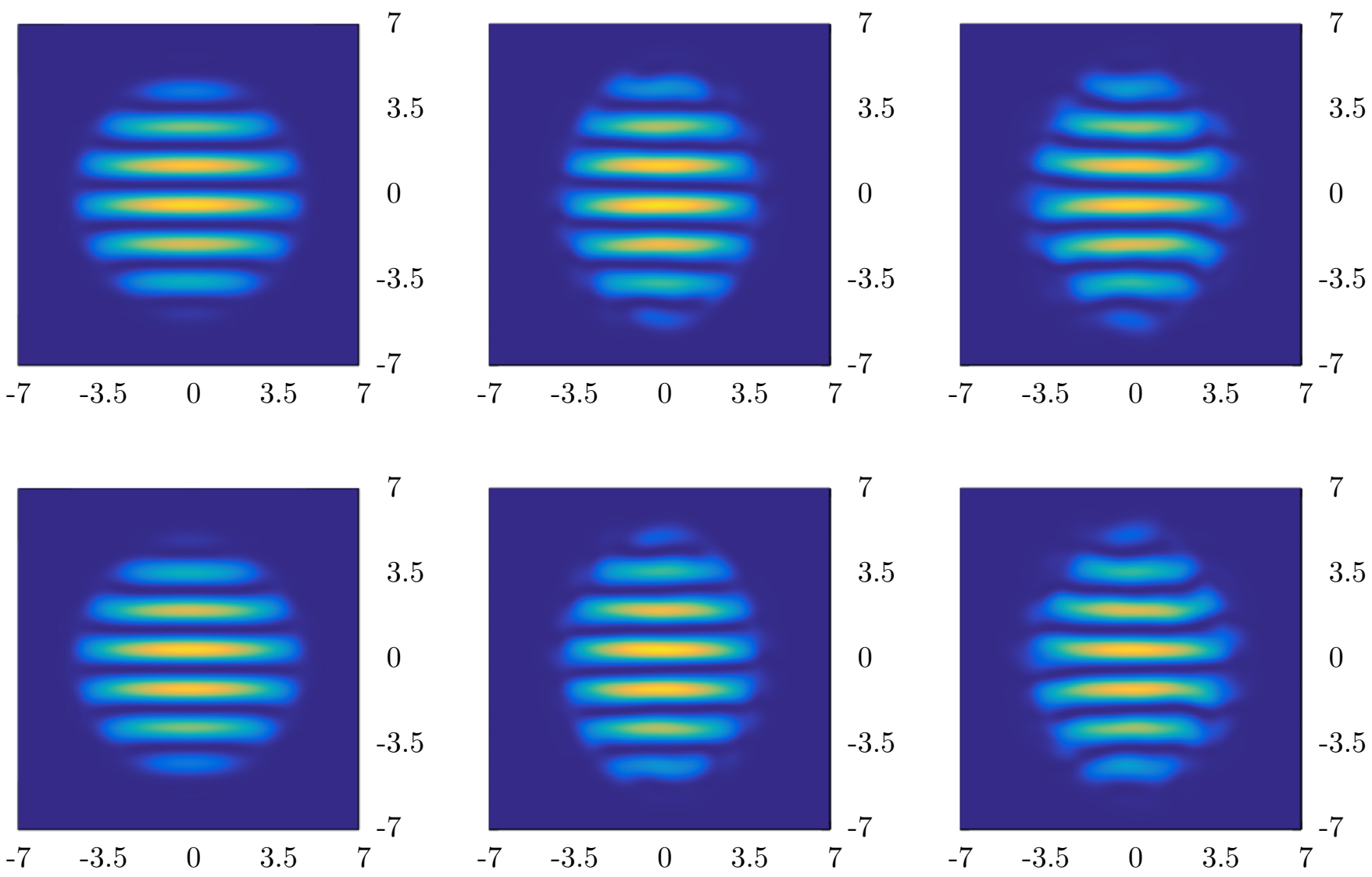

Figure 6: Example 3 (with $\Omega=0$ ): density functions $\left|\psi_{1}\right|^{2}$ (top) and $\left|\psi_{2}\right|^{2}$ (bottom) (on $]-7 ; 7\left[^{2}\right.$ ) at times $t=0$ (left), $t=4$ (center) and $t=T_{\mathrm{f}}$ (right).
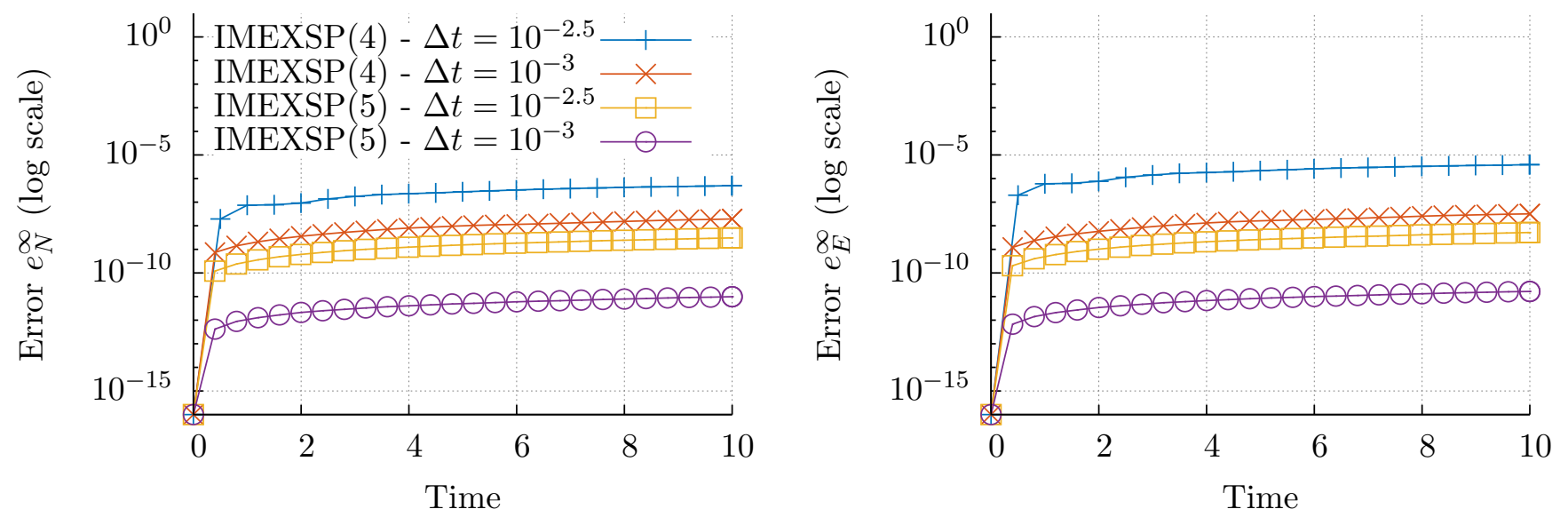

Figure 7: Example 3 (with $\Omega=0$ ): evolution of $e_{N}^{n}$ (left) and $e_{E}^{n}$ (right) for the $\operatorname{IMEXSP}(4)$ and $\operatorname{IMEXSP}(5)$ with $\Delta t=10^{-2.5}, 10^{-3}$.

with $\tau=10^{-6}, 10^{-10}$. Firstly, we observe that $\operatorname{IMEXSP}(4) 3$ attains the a priori fixed accuracy on the energy (since the variable technique is based on the energy indicator) for $\tau=10^{-10}$ while for $\tau=10^{-6}$ a slight deterioration appears. Let us point out that the two schemes achieve the same levels of accuracy for given time steps. Indeed, IMEXSP(4)3 with $\tau=10^{-10}$ runs with a time step $\Delta t \approx 10^{-3.4}$ (see Table 2 and Figure 11 ) and leads to a plot which is very close to the one obtained by IMEXSP(4) with $\Delta t=10^{-3.5}$. We thus conclude that the adaptive time step strategy is very robust and accurate. 

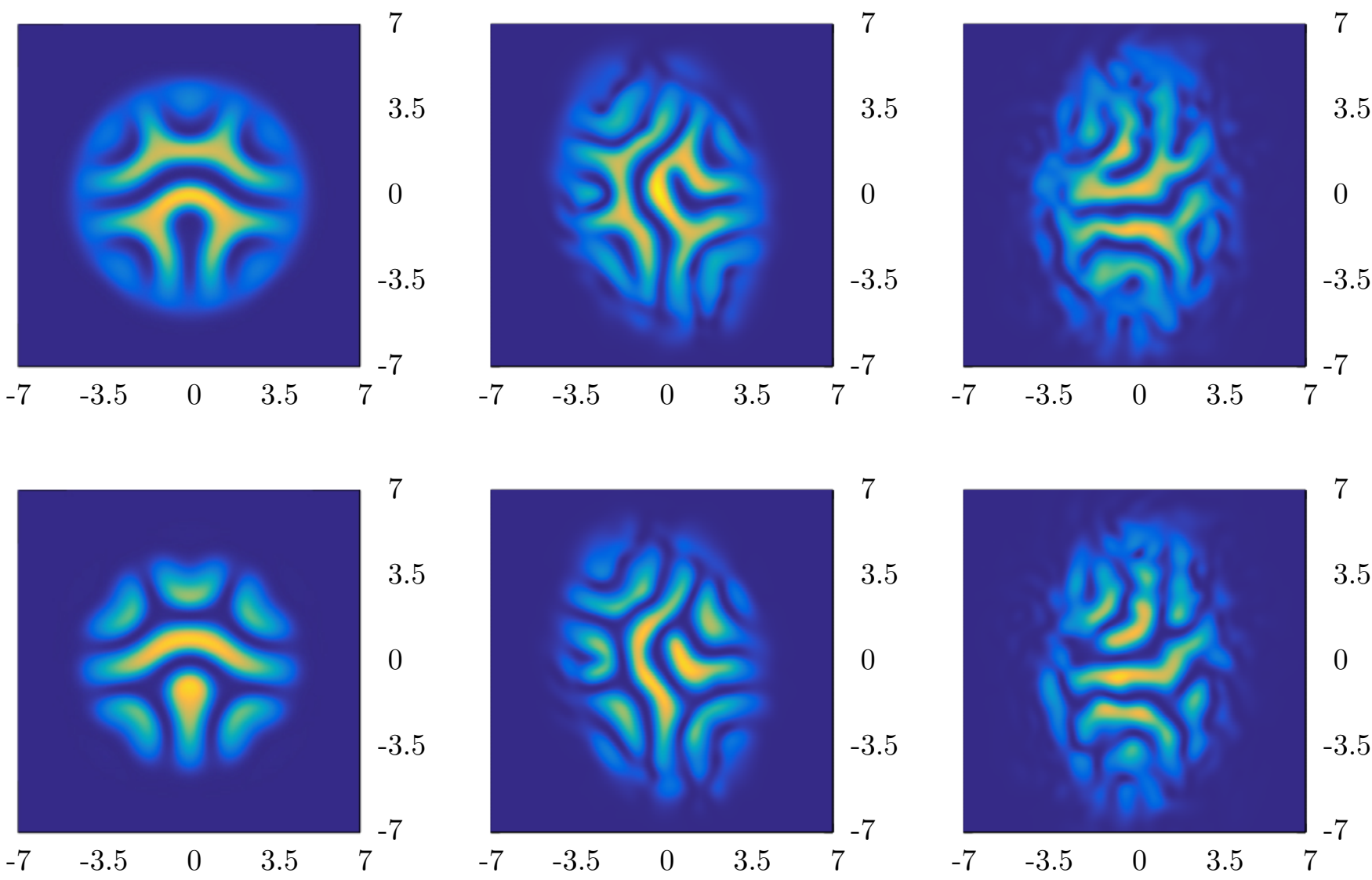

Figure 8: Example 3 (with $\Omega=0.4$ ): density functions $\left|\psi_{1}\right|^{2}$ (top) and $\left|\psi_{2}\right|^{2}$ (bottom) (on ] - 7; 7 [2) at times $t=0$ (left), $t=4$ (center) and $t=T_{\mathrm{f}}$ (right).
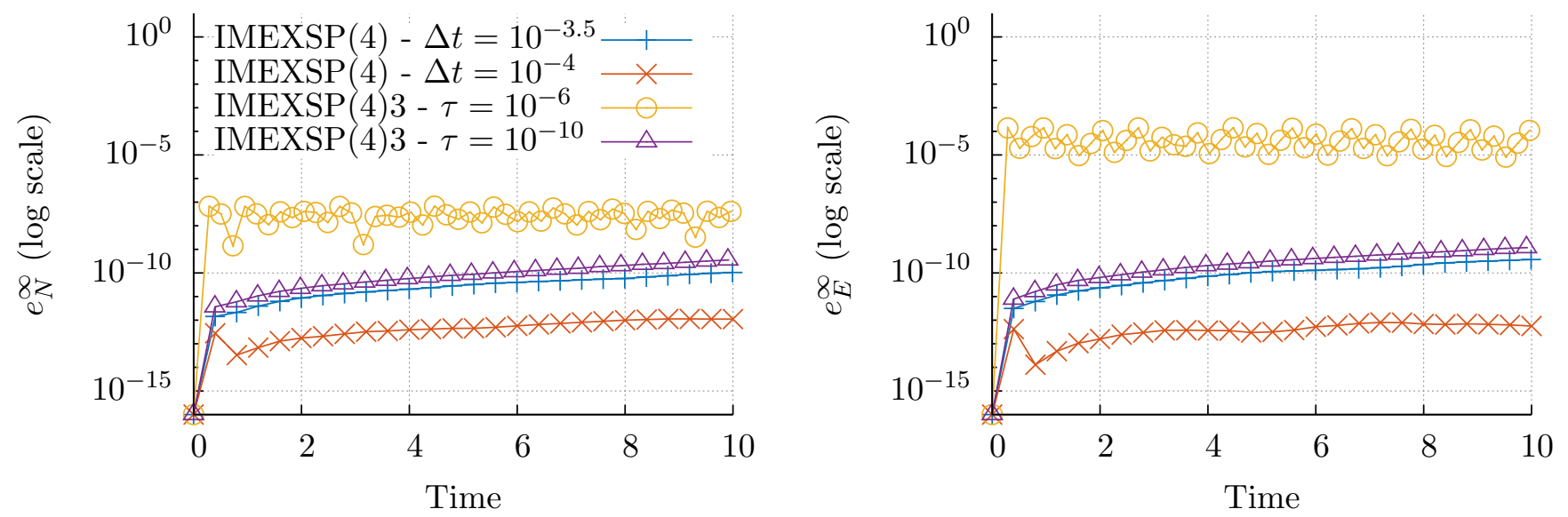

Figure 9: Example 3 (with $\Omega=0.4$ ): evolution of $e_{N}^{n}$ (left) and $e_{E}^{n}$ (right) for the $\operatorname{IMEXSP}(4)($ with $\Delta t=$ $10^{-3.5}, 10^{-4}$ ) and $\operatorname{IMEXSP}(4) 3$ (with $\tau=10^{-6}, 10^{-10}$ ) schemes.

We report in Figure 10 the same plots but for the fifth-order schemes, i.e. $\operatorname{IMEXSP}(5)$ and $\operatorname{IMEXSP}(5) 4$. We can see that in this case the constraints on the energy are perfectly satisfied for $\tau=10^{-6}$ and $\tau=10^{-10}$. Moreover, we point out that the variable time step schemes are very robust since they always give accurate results. For instance, we observe a loss of precision for the fixed strategy with $\Delta t=10^{-3}$.

We report the number of iterations in Table 2 (which also corresponds to the computational cost) with respect to the time step for the fixed schemes and the accuracy threshold $\tau$ for the adaptive techniques. In the 

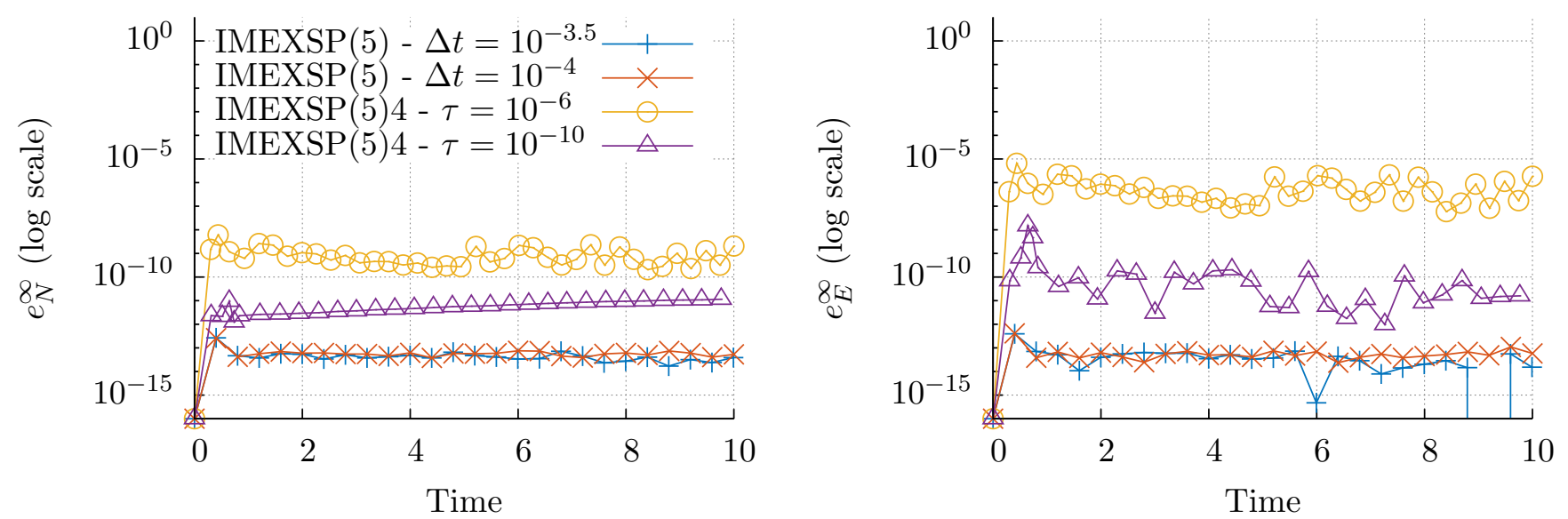

Figure 10: Example 3 (with $\Omega=0.4$ ): evolution of $e_{N}^{n}$ (left) and $e_{E}^{n}$ (right) for the IMEXSP(5) (with $\Delta t=$ $10^{-3.5}, 10^{-4}$ ) and $\operatorname{IMEXSP}(5) 4$ (with $\tau=10^{-6}, 10^{-10}$ ) schemes.

latter case, we also list the approximate values of the time steps $\Delta t$ (whose complete evolutions can be seen in Figure 11) used by the adaptive time step technique for $\tau=10^{-6}$ and $\tau=10^{-10}$. We also provide the number (\#) and percentage (\%) of rejections involved in the Algorithm 1. We remark that using a high-order scheme is crucial for efficiency reasons. Even if the cost-per-iteration of the fifth-order scheme is larger than for the fourth-order one (i.e. 8 and 6 stages are used, respectively), it is clear that IMEXSP(4)3 leads to some accurate results but IMEXSP(5)4 is nevertheless more accurate and efficient. The number of rejections per iteration in Algorithm 1 is generally zero and the percentage of rejections is negligible. This confirms that the adaptive time stepping strategy is realized at almost no additional cost. Moreover, it is interesting to notice that the IMEXSP (5) scheme with constant time step $\Delta t=10^{-3}$ blows up before reaching the final time (we do not report the results here). Thus, the very small difference in the time step detected by the adaptive scheme is central. This emphasizes on the fact that having a robust adaptive time stepping strategy is extremely important for this kind of situation, ensuring the stability of the schemes as well as the accurate computation of the wave functions and macroscopic quantities.

\begin{tabular}{|l|l|c|c|c|c|}
\hline Scheme & $\Delta t$ & $\tau$ & \# iterations & \#rejections & $\%$ rejections \\
\hline \hline IMEXSP $(4)$ & $10^{-3.5}\left(\approx 3.2 \times 10^{-4}\right)$ & & 31623 & - & - \\
& $10^{-4}$ & & 100000 & - & - \\
\hline IMEXSP $(4) 3$ & $10^{-3.37}\left(\approx 4.3 \times 10^{-4}\right)$ & $10^{-6}$ & 23813 & 1224 & $5.1 \%$ \\
& $10^{-3.4}\left(\approx 3.9 \times 10^{-4}\right)$ & $10^{-10}$ & 25242 & 1 & $4 \times 10^{-3} \%$ \\
\hline IMEXSP $(5)$ & $10^{-3.5}\left(\approx 3.2 \times 10^{-4}\right)$ & & 31623 & - & - \\
& $10^{-4}$ & & 100000 & - & - \\
\hline IMEXSP $(5) 4$ & $10^{-3.06}\left(\approx 8.7 \times 10^{-4}\right)$ & $10^{-6}$ & 11689 & 308 & $2.6 \%$ \\
& $10^{-3.07}\left(\approx 8.6 \times 10^{-4}\right)$ & $10^{-10}$ & 12618 & 350 & $2.8 \%$ \\
\hline
\end{tabular}

Table 2: Example 3 (with $\Omega=0.4$ ): numerical results for the various schemes (orders, time step strategies).

We report the evolution of the time step according to the time iterations on Figure 11 . We remark that small changes in the time step may give rise to sensibly different results in accuracy. Moreover, for instance concerning the fifth-order IMEXSP(5) scheme, one achieves the same accuracies by using $\Delta t=10^{-3.5}$ and $\Delta t=10^{-4}$, while the first case leads to the most efficient solution. In this context, we clearly see how robust and efficient the variable time step technique is. We also observe that the fifth-order scheme needs a larger time step than the fourth-order one, resulting in a lower total computational cost even if the cost-per-iteration is larger.

Let us recall that other tableaux than the one proposed here could be chosen. For example, we present some 


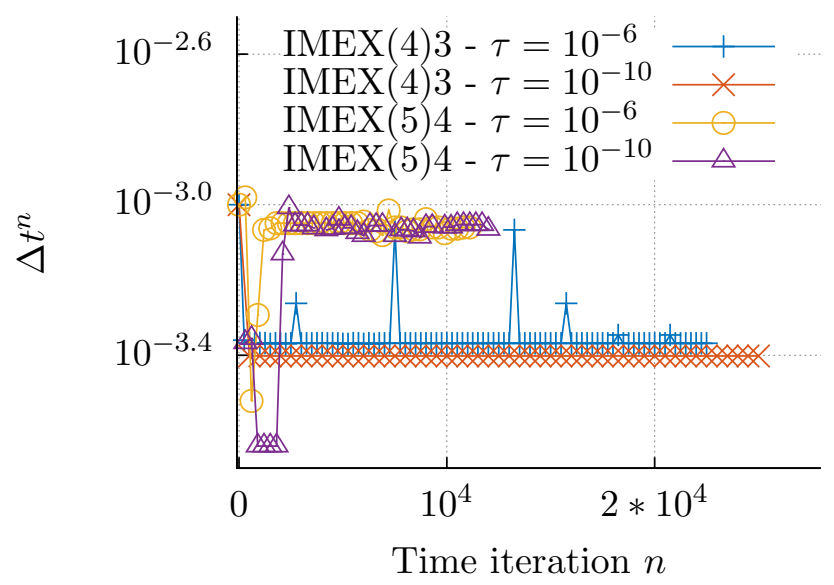

Figure 11: Example 3 (with $\Omega=0.4$ ): time step variations for the $\operatorname{IMEXSP}(4) 3$ and $\operatorname{IMEXSP}(5) 4$ schemes (in $\log$ scale) with $\tau=10^{-6}, 10^{-10}$ at each iteration ( $x$-axis) up to final time.

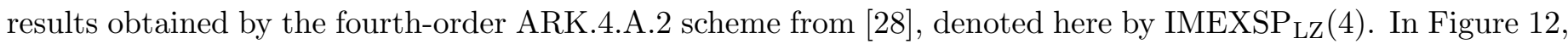
we plot the error on the mass and energy for this scheme. We observe that it yields very accurate results and that it has, in this case, some better stability properties than IMEXSP(4). However, we point out that small values of $\Delta t$ are necessary to reach a high accuracy. We compare in Figure 13 these two fourth-order schemes and see that they achieve the same maximal accuracy. Moreover, we observe that the $\operatorname{IMEXSP}_{\mathrm{LZ}}(4)$ scheme provides a higher precision with a fixed time step but for 7 stages (while IMEXSP(4) needs 6 stages). Finally, they are equivalent in this case concerning the efficiency.
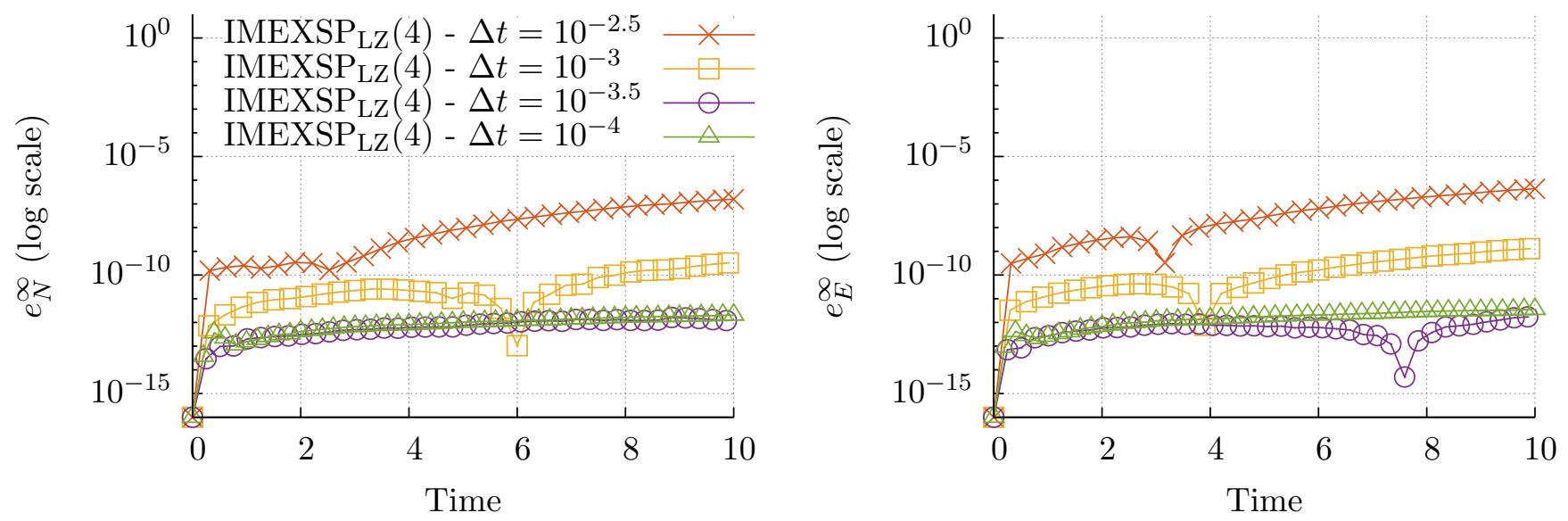

Figure 12: Example 3 (with $\Omega=0.4$ ): evolution of $e_{N}^{n}$ (left) and $e_{E}^{n}$ (right) for the $\operatorname{IMEXSP}_{\mathrm{LZ}}(4)$ scheme with $\Delta t=10^{-2}, 10^{-2.5}, 10^{-3}, 10^{-3.5}, 10^{-4}$.

\section{Conclusion}

We have presented highly accurate numerical schemes for computing the dynamics of general multi-components systems of Gross-Pitaevskii equations. The method is based on the use of high-order IMplicit-EXplicit (IMEX) schemes in time and a Fourier pseudo-spectral approximation in space. The resulting IMEXSP schemes are shown to be fully explicit in practice. Even if they are not exactly mass nor energy conserving, they however conserve these quantities with a high accuracy. In addition, the schemes are easy to implement and robust if they are combined with an adaptive time stepping strategy. In particular, IMEXSP schemes are an interesting alternative to TSSP schemes that may be difficult to design with a high-order of precision in some physically 

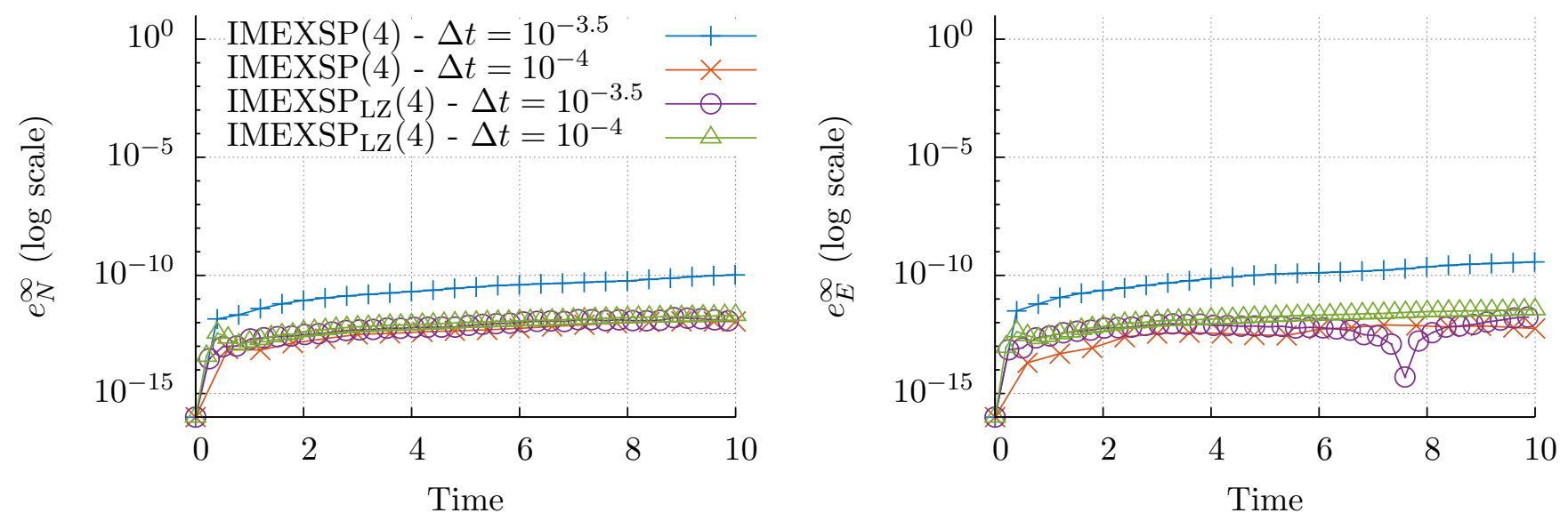

Figure 13: Example 3 (with $\Omega=0.4$ ): comparison of $e_{N}^{n}$ (left) and $e_{E}^{n}$ (right) for the $\operatorname{IMEXSP}(4)$ and $\operatorname{IMEXSP}_{\mathrm{LZ}}(4)$ schemes with $\Delta t=10^{-3.5}, 10^{-4}$.

relevant situations (e.g. for non-autonomous systems or for very general systems of GPE). The IMEXSP schemes are compared with TSSP schemes for solving autonomous GPE and numerically tested for a two-components non-rotating/rotating spin-orbit coupled GPE that arises in the modeling of BEC.

Acknowledgments. The authors thank the support of the french ANR grant ANR-12-MONU-0007-02 BECASIM (Modèles Numériques call).

\section{References}

[1] A. Aftalion and P. Mason. Phase diagrams and Thomas-Fermi estimates for spin-orbit-coupled Bose-Einstein condensates under rotation. Phys. Rev. A, 88:023610, Aug 2013.

[2] M.H. Anderson, J.R. Ensher, M.R. Matthews, C.E. Wieman, and E.A. Cornell. Observation of Bose-Einstein condensation in a dilute atomic vapor. Science, 269(5221):198-201, Jul 141995.

[3] X. Antoine, W. Bao, and C. Besse. Computational methods for the dynamics of the nonlinear Schrödinger/Gross-Pitaevskii equations. Computer Physics Communications, 184(12):2621-2633, 2013.

[4] X. Antoine and R. Duboscq. GPELab, a Matlab toolbox to solve Gross-Pitaevskii equations I: Computation of stationary solutions. Computer Physics Communications, 185(11):2969-2991, 2014.

[5] X. Antoine and R. Duboscq. Robust and efficient preconditioned Krylov spectral solvers for computing the ground states of fast rotating and strongly interacting Bose-Einstein condensates. J. Comput. Phy., 258:509-523, 2014.

[6] X. Antoine and R. Duboscq. GPELab, a Matlab toolbox to solve Gross-Pitaevskii equations II: Dynamics and stochastic simulations. Computer Physics Communications, 193:95-117, 2015.

[7] X. Antoine and R. Duboscq. Modeling and Computation of Bose-Einstein Condensates: Stationary States, Nucleation, Dynamics, Stochasticity. In Besse, C and Garreau, JC, editor, Nonlinear Optical and Atomic Systems: at the Interface of Physics and Mathematics, volume 2146 of Lecture Notes in Mathematics, pages 49-145. 2015.

[8] P. Antonelli, D. Marahrens, and C. Sparber. On the Cauchy problem for nonlinear Schrödinger equations with rotation. Discrete Contin. Dyn. Syst., 32(3):703-715, 2012.

[9] U.M. Ascher, S.J. Ruuth, and R.J. Spiteri. Implicit-explicit Runge-Kutta methods for time-dependent partial differential equations. Appl. Numer. Math., 25(2-3):151-167, 1997. Special issue on time integration (Amsterdam, 1996).

[10] W. Bao and Y. Cai. Mathematical theory and numerical methods for Bose-Einstein condensation. Kinetic and Related Models, 6(1):1-135, 2013.

[11] W. Bao, D. Marahrens, Q. Tang, and Y. Zhang. A simple and efficient numerical method for computing the dynamics of rotating Bose-Einstein condensates via rotating Lagrangian coordinates. SIAM J. Scientific Computing, 35(6), 2013.

[12] W. Bao and H. Wang. An efficient and spectrally accurate numerical method for computing dynamics of rotating Bose-Einstein condensates. J. Comput. Physics, 217(2):612-626, 2006.

[13] J. Belmonte-Beitia and J. Cuevas. Solitons for the cubic-quintic nonlinear Schrödinger equation with time- and space-modulated coefficients. J. Phys. A, 42(16):165201, 11, 2009. 
[14] C. Besse, G. Dujardin, and I. Lacroix-Violet. High order exponential integrators for nonlinear Schrödinger equations with application to rotating Bose-Einstein condensates. arXiv preprint arXiv:150\%.00550, 2015.

[15] S. Blanes, F. Casas, and A. Murua. Splitting methods in the numerical integration of non-autonomous dynamical systems. Rev. R. Acad. Cienc. Exactas Fi s. Nat. Ser. A Math. RACSAM, 106(1):49-66, 2012.

[16] S. Blanes and P. C. Moan. Practical symplectic partitioned Runge-Kutta and Runge-Kutta-Nyström methods. J. Comput. Appl. Math., 142(2):313-330, 2002.

[17] J.C. Butcher. The Numerical Analysis of Ordinary Differential Equations. Wiley, Chichester, 1987.

[18] J.C. Butcher. A history of Runge-Kutta methods. Appl. Numer. Math., 20(3):247-260, 1996. Selected keynote papers presented at 14th IMACS World Congress (Atlanta, GA, 1994).

[19] M.H. Carpenter and C.A. Kennedy. Fourth-order 2N-storage Runge-Kutta schemes. Nasa tm, 109112:871-885, 1994.

[20] F. Castella, P. Chartier, S. Descombes, and G. Vilmart. Splitting methods with complex times for parabolic equations. BIT, 49(3):487-508, 2009.

[21] S. Descombes, M. Duarte, T. Dumont, V. Louvet, and M. Massot. Adaptive time splitting method for multi-scale evolutionary partial differential equations. Confluentes Math., 3(3):413-443, 2011.

[22] G. Dimarco, L. Mieussens, and V. Rispoli. An asymptotic preserving automatic domain decomposition method for the VlasovPoisson-BGK system with applications to plasmas. J. Comput. Phys., 274:122-139, 2014.

[23] G. Dimarco, L. Pareschi, and V. Rispoli. Implicit-explicit Runge-Kutta schemes for the Boltzmann-Poisson system for semiconductors. Commun. Comput. Phys., 15(5):1291-1319, 2014.

[24] A.L. Fetter. Vortex dynamics in spin-orbit-coupled Bose-Einstein condensates. Physical Review A, 89(2):023629, 2014.

[25] E. Hairer, C. Lubich, and G. Wanner. Geometric numerical integration: structure-preserving algorithms for ordinary differential equations, volume 31. Springer Science \& Business Media, 2006.

[26] K. Kasamatsu. Dynamics of quantized vortices in Bose-Einstein condensates with laser-induced spin-orbit coupling. Physical Review A, 92(6):063608, 2015.

[27] C.A. Kennedy and M.H. Carpenter. Additive Runge-Kutta schemes for convection-diffusion-reaction equations. Applied Numerical Mathematics, 44(1):139-181, January 2003.

[28] H. Liu and J. Zou. Some new additive Runge-Kutta methods and their applications. J. Comput. Appl. Math., 190(1-2):74-98, 2006.

[29] W.X. Ma and M. Chen. Direct search for exact solutions to the nonlinear Schrödinger equation. Appl. Math. Comput., 215(8):2835-2842, 2009.

[30] P. Mason and A. Aftalion. Classification of the ground states and topological defects in a rotating two-component Bose-Einstein condensate. Physical Review A, 84(3):033611, 2011.

[31] J. Ming, Q. Tang, and Y. Zhang. An efficient spectral method for computing dynamics of rotating two-components Bose-Einstein condensates via coordinate transformation. J. Comput. Phys., 258:538-554, 2014.

[32] C. Qu, K. Sun, and C. Zhang. Quantum phases of Bose-Einstein condensates with synthetic spin-orbital-angular-momentum coupling. Physical Review A, 91(5):053630, 2015.

[33] M. Seydaoğlu and S. Blanes. High-order splitting methods for separable non-autonomous parabolic equations. Appl. Numer. Math., 84:22-32, 2014.

[34] L.F. Shampine. Error estimation and control for ODEs. J. Sci. Comput., 25(1):3-16, 2005.

[35] L. Wen and J. Li. Structure and dynamics of a rotating superfluid Bose-Fermi mixture. Physical Review A, 90(5):053621, 2014.

[36] H. Yoshida. Construction of higher order symplectic integrators. Phys. Lett. A, 150(5-7):262-268, 1990.

[37] H. Zhai. Degenerate quantum gases with spin-orbit coupling: a review. Rep. Progr. Phys., 78(2):026001, $25,2015$. 